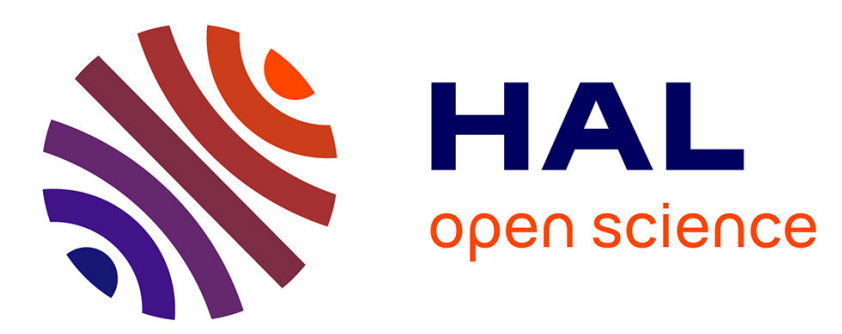

\title{
Kinematics and performance analysis of a novel concentric tube robotic structure with embedded soft micro-actuation
}

\author{
Mohamed Taha Chikhaoui, Kanty Rabenorosoa, Nicolas Andreff
}

\section{- To cite this version:}

Mohamed Taha Chikhaoui, Kanty Rabenorosoa, Nicolas Andreff. Kinematics and performance analysis of a novel concentric tube robotic structure with embedded soft micro-actuation. Mechanism and Machine Theory, 2016, 104, pp.234-254. hal-02868186

\section{HAL Id: hal-02868186 \\ https://hal.science/hal-02868186}

Submitted on 15 Jun 2020

HAL is a multi-disciplinary open access archive for the deposit and dissemination of scientific research documents, whether they are published or not. The documents may come from teaching and research institutions in France or abroad, or from public or private research centers.
L'archive ouverte pluridisciplinaire HAL, est destinée au dépôt et à la diffusion de documents scientifiques de niveau recherche, publiés ou non, émanant des établissements d'enseignement et de recherche français ou étrangers, des laboratoires publics ou privés. 


\title{
Kinematics and performance analysis of a novel concentric tube robotic structure with embedded soft micro-actuation
}

\author{
Mohamed Taha Chikhaoui, Kanty Rabenorosoa, and Nicolas Andreff \\ AS2M Department, FEMTO-ST Institute \\ 24 rue Alain Savary - 25000 Besançon, France
}

\begin{abstract}
Continuum robots have shown astounding abilities to assist surgeons reaching confined spaces in the human body. Thus, accurate control of these manipulators, and particularly concentric tube robots, is required in order to achieve intracorporeal microrobotic interventions. We present hereby an improvement of this kinematic structure based on embedded soft micro-actuators. Two models for single and double direction curvature control are introduced. We demonstrate that kinematics are enhanced with respect to the standard approach in terms of holonomy, actuation redundancy and workspace covering. Further kinematic analysis enables the detection of singular configurations. The number of the end-effector pose occurrences that can be reached in a given volume (one cubic millimeter) are computed as well. Finally, the advantages of the novel structures are proven using performance indices.
\end{abstract}

Keywords: Continuum robot, kinematics, holonomy, redundancy, soft micro-actuators, intracorporeal microrobotics.

\section{Introduction}

Observing the wondrous abilities of some natural creatures has always been motivating as well as inspiring, particularly for researchers. Concerning soft structures, one of the most fascinating is the elephant's trunk. Notwithstanding its flexibility, which would trivially mean its weakness, that appendage is able at one moment to show great strength and power when lifting heavy loads as tree trunks. It can also present delicacy and precision when eating or interacting with their calves. Figure 1 shows elephants at almost the same trunk configuration performing different tasks with varied effort and stiffness requirements.

\subsection{Continuum robots}

One can distinguish the continuum robots from the "traditional" hard robots with the absence of rigid links and joints for the latter. Typically, the former presents a distributed deformation

Email addresses: mohamed.chikhaoui@femto-st.fr (), kanty.rabenorosoa@femto-st.fr (), nicolas.andreff@femto-st.fr () 
a)

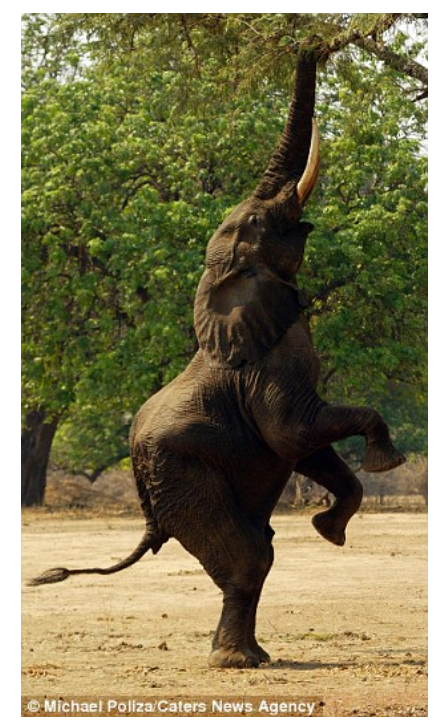

b)

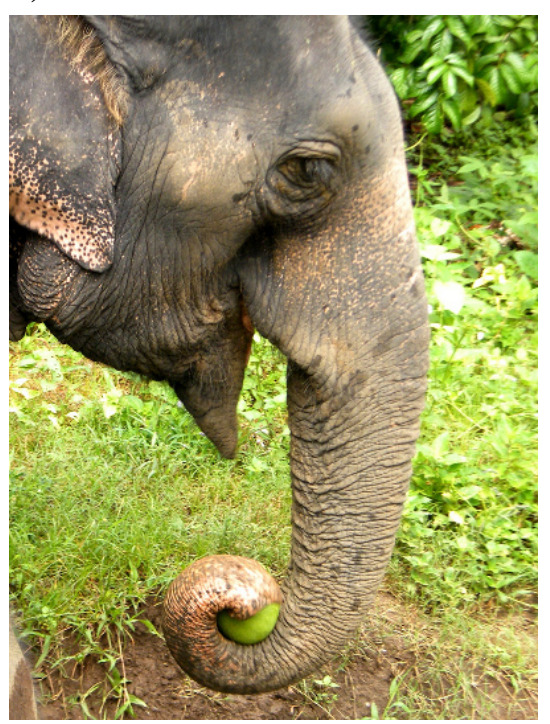

Figure 1: Almost the same position and shape of an elephant trunk a) wresting a tree branch, which requires high forces (Michael Poliza / Caters News Agency (C)2011), and b) picking a mango with the appropriate delicacy (Josh Urich / Canva Photographers (C)2015).

performed by scattered actuators throughout the structure. Trivedi et al. (2008) suggested a different approach, classifying them into a subset of hyper-redundant robots. However, the difference between hyper-redundant and continuum manipulators is subtle. Thus, it is challenging to provide a clear classification. Hyper-redundancy implies the presence of discrete elements within the backbone. The continuity of the robot shape, regardless of its structure, is not necessarily the suitable criterion to consider a robot as continuum. For instance, early prototypes including snake-like robots developed by Hirose (1993) and elephant trunk robots developed by Walker and Hannan (1999) present a continuous shape but they are considered hyper-redundant robots. These robots are considered as bio-inspired as they mimic animal parts or movements. Observing the abilities and the performances of hydroskeletons and muscular hydrostats, other bio-inspired prototypes have been developed later. One can cite the starfish-like gel robot developed by Otake et al. (2002), octopus-like robot (OctArm) developed by Jones and Walker (2006b), elephant trunk-like robot developed by Wolf et al. (2003), and snake-like robot developed by Simaan et al. (2004). The latter brought an enthralling classification. It is based, notably, on the actuation technique, whether it is continuous or discrete. In order to actuate continuum robots, different techniques have been used, such as

- cables along the backbone, equally distributed around the tubular robot diameter as proposed by Anderson and Horn (1967) depicted in Figure 2a, Ciéslak and Morecki (1999); Hannan and Walker (2003); Gravagne et al. (2003) and Camarillo et al. (2008) depicted in Figure 2b or elastic rods to raise the rigidity of the robot to permit transmission of compressive forces as used by Simaan et al. (2004); Xu and Simaan (2008); Rone and Ben-Tzvi (2014),

- fluidic (pneumatic and hydraulic) actuators by Bailly and Amirat (2005); Chen (2005); Jones and Walker (2006a,b); Ikeuchi and Ikuta (2009); Cianchetti et al. (2013), 
- Shape Memory Alloy (SMA) based actuators by Ikuta et al. (1988); Lee et al. (2002); Langelaar and van Keulen (2004); Haga et al. (2010),

- Electro-Active Polymer (EAP) based actuators by Bar-Cohen (2001); Choi et al. (2002); Kim and Tadokoro (2007); Shoa et al. (2008); Lee et al. (2009), and

- concentric tube robots depicted in Figure 2c by Webster III et al. (2009, 2013); Dupont et al. (2010, 2012b).

a)

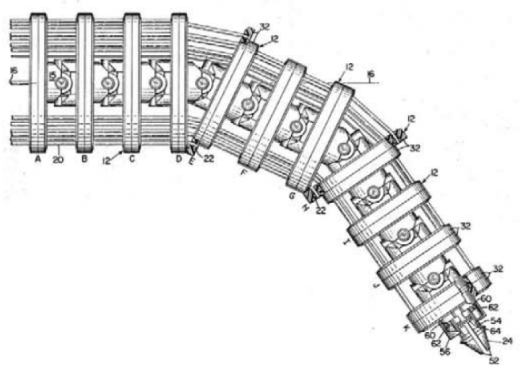

b)

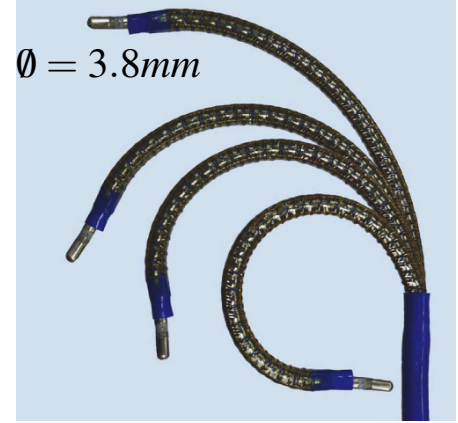

c)

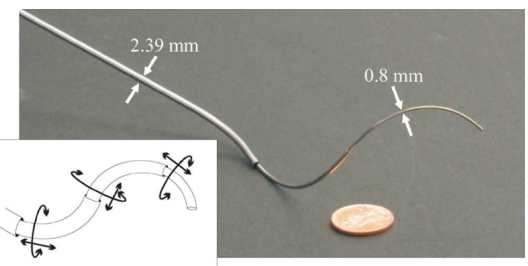

Figure 2: a) The Tensor Arm of Anderson and Horn (1967), considered as the continuum robot ancestor. b) The Hansen Medical Sensei ${ }^{\circledR}$ system described mechanically and kinematically by Camarillo et al. (2008). c) The concentric tube robot developed by Webster III et al. (2009).

\subsection{Modeling continuum robots}

Modeling continuum robots is slightly more challenging than modeling traditional robots due to the lack of rigid links. For the latter, link lengths and joint angles are substantially used to register the pose of the robot and define its kinematics. Consequently, for the former, a wide diversity of models, formalisms, and coordinate frame choices have been proposed during the last 50 years. They vary from the early work of Anderson and Horn (1967)- considered as the first example of hyper-redundant robot - to the following developments outlined in several reviews such as those introduced by Hirose (1993); Robinson and Davies (1999); Webster III and Jones (2010). In order to predict the behavior of continuum manipulators, models are typically based on elasticity considerations. To summarize, two major models were introduced. One is the Theory of Cosserat rod, introduced within this topic by Jones et al. (2009); Rucker and Webster III (2011); Dehghani and Moosavian (2011). While it provides precise modeling of continuum robots using differential equations, this method lacks stability concerning the dynamic model and does not provide a closedform formulation, Dehghani and Moosavian (2013). The other method is based on piece-wise constant curvature assumption and can alleviate many of these concerns. It provides direct and inverse kinematic modeling, with closed-form solutions. Chirikjian (1993) drew the foundations for kinematic theory of hyper-redundant robots which led later to the work of Gravagne and Walker. Gravagne and Walker (2000b) started modeling the kinematics of planar continuum manipulators for one section with one degree of freedom (DoF), then Gravagne and Walker (2000a) extended 
it to two sections. Further developments by Gravagne and Walker $(2001,2002)$ led to kinematic Jacobian computation, with manipulability and force analysis. A modified Denavit-Hartenbergtype approach was introduced by Jones and Walker (2005) to model a two-sections continuum robot with six degrees of mobility. Other derivations were also used as Frenet-Serret frames by Hannan and Walker (2003), integral formulation by Chirikjian and Burdick (1994), and exponential coordinates by Sears and Dupont (2006); Webster III et al. (2006a). These diverse formalisms converge to the same final result under the constant curvature assumption, as demonstrated by Webster III and Jones (2010).

\subsection{Concentric tube robots}

Our interest is brought on concentric tube robots for surgical applications due to their high miniaturization potential and their ability to describe miscellaneous shapes. Their significant mobility permits to navigate through complex anatomy and perform surgical tasks. They are constituted of several curved and concentric telescopic tubes that can either translate or rotate along their principal axes. Thus, they can be steered along a curved path through the tube depending on elastic interactions, and house tools within their lumen in order to achieve diagnosis or intervention.

Concentric tube robots promise astounding abilities, particularly in medical applications under strong dimensional constraints (diameter below few millimeters). Potential or under-development applications include endonasal skull base surgery introduced by Burgner et al. (2011), intracerebral hemorrhage evacuation by Burgner et al. (2013), surgical resection of the hippocampus by Comber et al. (2012), transurethral laser prostate surgery by Hendrick et al. (2014), surgery in otolaryngology by Schneider et al. (2013), bronchoscopy operations by Torres et al. (2012), all developed within R. J. Webster's research group; neurosurgery and endoscopy by Anor et al. (2011); Butler et al. (2012); Dupont et al. (2012a); Bergeles and Dupont (2013), beating-heart closure of atrial septal defects by Bedell et al. (2011), and suturing needle by Sears and Dupont (2006) all developed within P. Dupont's research group.

\subsection{Proposed embedded micro-actuation for concentric tube robots}

The major target of this work is to improve this promising technique of concentric tubes by adding variable and controlled curvature to the tubes using embedded micro-actuation based on active soft materials, namely Electro-Active Polymers (EAP). Indeed, significant results have been demonstrated by Shoa et al. (2008) using the conjugated ionic polymer PolyPyrrole (PPy). Adding to its biocompatibility, PPy is distinguished by its lightweight and the very low activation voltages required (less than one volt) without any additional heating, in contrast with SMA and Piezoelec-

tric materials. Furthermore, it provides high curvature control and may be grafted on very small diameters (down to $1 \mathrm{~mm}$ ). These features are summarized in Table 1. 


\begin{tabular}{l||c|c|c}
\multicolumn{4}{c}{ Table 1: Qualitative comparison of different actuation techniques } \\
Material & Shape Memory Alloys & Piezoelectric & Electro-Active Polymers \\
\hline Biocompatibility & high & low & high \\
Size & small & small & very small $(\sim 10-40 \mu \mathrm{m})$ \\
Strain & average & low & average $(\sim 20 \%)$ \\
Activation voltage & high & very high & very low $(<2 \mathrm{~V})$ \\
Dynamics & average & high & average $(\sim 5$ seconds $)$
\end{tabular}

The embedded actuators are developed as thin antagonistic electrodes coated around the tube as described in Figure 3a,b for single and double bending direction respectively. The actuation of every couple of electrodes with opposed voltages (of less than one volt for PPy) produces a bending moment around the transversal axis as shown in Figure $3 \mathrm{c}$ described in details by Shoa et al. (2010), among others.
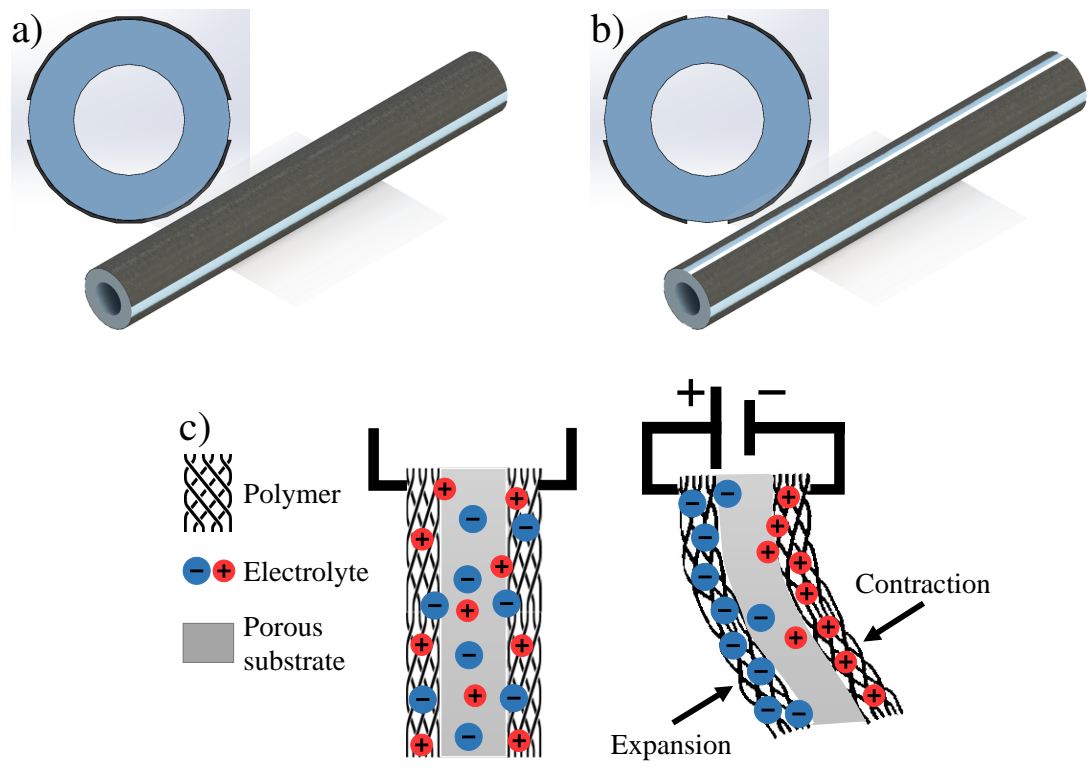

Figure 3: Design of PPy-based micro-actuators of approximately $30 \mu \mathrm{m}$ patterned on a $1 \mathrm{~mm}$ diameter tube as: a) one pair of antagonistic electrodes permitting one bending direction, and b) two pairs of antagonistic electrodes enabling double bending direction. The insets display a cross-sectional view showing the thinness of the PPy layer. c) Actuation principle of the PPy layers: a strain is produced when the antagonistic electrodes are electrically activated with opposite voltages. The expansion of the positive electrode faces the contraction of the negative one due to the insertion and the removal of ions respectively.

Adding to that, using four electrodes (cf. Figure 3b) is potentially useful to decrease the actuation unit volume, knowing the consequent size of the rotation modules used in both Webster's (cf. Figure 4a) and Dupont's works. In contrast, the PPy-based actuators to use are only about $30 \mu \mathrm{m}$ in thickness, as proposed by Shoa et al. (2008). This induces a dimensional increase of only $3 \%$ for a $1 \mathrm{~mm}$ tube diameter for instance. The actuators used by Shoa et al. (2008) produced enough force to bend a catheter of $15 \mathrm{~mm}$ to $100 \mathrm{~m}^{-1}$ curvature (approximately $90^{\circ}$ bending angle). Alici 
and Huynh (2007) reported a force generation of up to $1.6 \mathrm{mN}$ on a planar micro-gripper. A design of our proposed structure is displayed in Figure 4b.

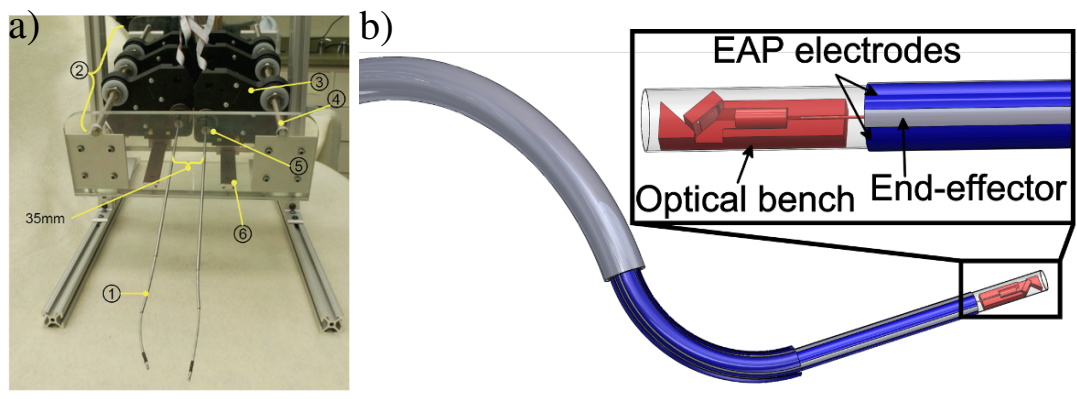

Figure 4: a) Actuation unit for three concentric tubes used by Burgner et al. (2011), b) CAD design of the embedded actuation principle presented by Chikhaoui et al. (2014b).

The main contribution of this paper is to develop geometric and kinematic models of concentric tube configurations with embedded PPy soft micro-actuation allowing curvature control. It extends the previous work introduced by Chikhaoui et al. (2014a). Further developments are carried out for generic continuum robot models with single and double direction curvature control and with more intense performance analysis. It is shown that this novel concept enables better conditioning of the kinematic Jacobian matrices and enhances manipulability. It provides also holonomy to the system and is able to produce redundancy. The concentric tube robotic structure is studied hereby for medical applications. We present a contactless scheme covering a wide range of medical applications whether for (i) diagnosis with housing a miniaturized camera, an optical bench (cf. Figure $4 \mathrm{~b}$ ), or an OCT probe or for (ii) intervention purpose with embedding a laser tool as demonstrated in Andreff and Tamadazte (2015) for laser micro-phonosurgery.

This article is organized as follows. The next section presents the developed geometric and kinematic models followed by their analysis. In order to demonstrate the enhancements produced by such a manipulator, the third section treats several numerical results concerning the workspace covered as well as the provided performances. Finally, the last section contains our conclusions and perspectives.

\section{Modeling}

In this section, the standard approach for modeling concentric tube robots under constant curvature assumption (CCM) is recalled. It describes the geometric model, as well as the independent and the specific kinematic Jacobian matrices. Furthermore, we will introduce the specific kinematic Jacobian matrices for two novel configurations: variable curvature (VCM) and double direction variable curvature (DDVCM). Afterwards, these models will be compared and analyzed in order to select the most theoretically reliable configuration.

\subsection{Standard approach}

The standard approach, detailed by Webster III (2007), assumes that for a concentric tube robot made of $n$ overlapping tubes, one can decompose the final assembly into $m$ successive links. 
Each link is assimilated to an arc of a circle. That arc can be perfectly described in the 3D space by three parameters. These parameters are consistent with the Denavit-Hartenberg formalism, Hannan and Walker (2003). The continuum robot joint parameters are called configuration space of arc parameters $(\kappa, \phi, \ell)$ where $\kappa$ is the curvature, $\phi$ is the arc equilibrium plane angle, and $\ell$ is the arc length. The relationship $\theta=\kappa \ell$ defines the bending angle of the arc. It can be introduced as an additional component that can be used for the parameterization. These parameters can be visualized in Figure 5 where the different frames that will be used later are defined.

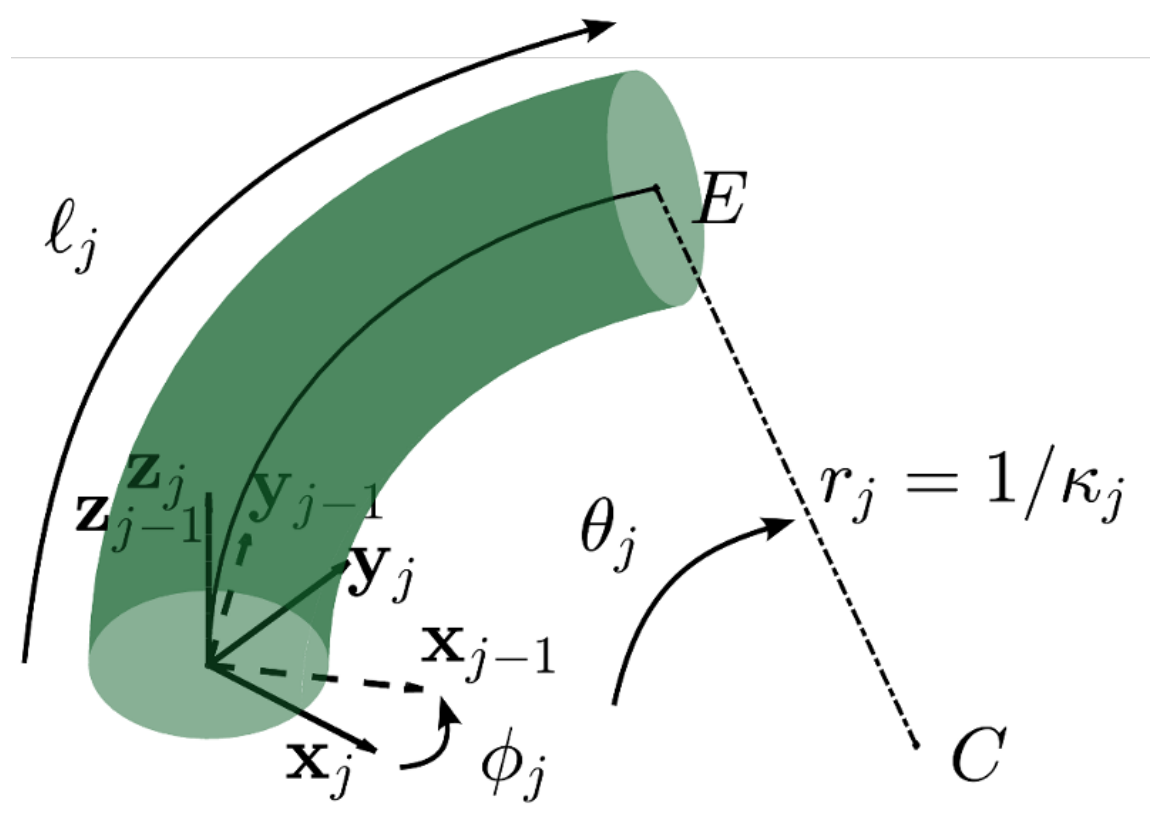

Figure 5: Schematic description of the configuration space parameters where the $z_{j}$-axis is defined as the current arc principal axis and $\phi_{j}$ describes the equilibrium plane angle of a link located in its $x-z$ plane. $r_{j}$ designates the arc radius where $\kappa_{j}=1 / r_{j}$. $\left\{x_{j-1}, y_{j-1}, z_{j-1}\right\}$ is the reference frame, located at the previous link end-effector.

\subsubsection{Geometric modeling}

A link is defined whenever the number of the overlapping tubes is modified or the shape of a tube is different (straight or curved). For example, if one curved tube $\left(L_{1}\right)$ is interacting with a second tube with smaller diameters constituted of a straight part $\left(L_{2}\right)$ and a curved part $\left(C_{2}\right)$, two links can be defined: the first for $L_{2}$ inside $C_{1}$, and the second for $C_{2}$ inside $C_{1}$. Considering Figure 6 , a concentric tube robot with $n=3$ tubes is sketched. Each tube, of a diameter $D_{i}$, consists of a straight proximal part $L_{i}$ and a curved distal part $C_{i}$. Considering the shape of the interacting parts of the tubes, $m=6$ sections can be defined, as sketched in Figure 6 . 


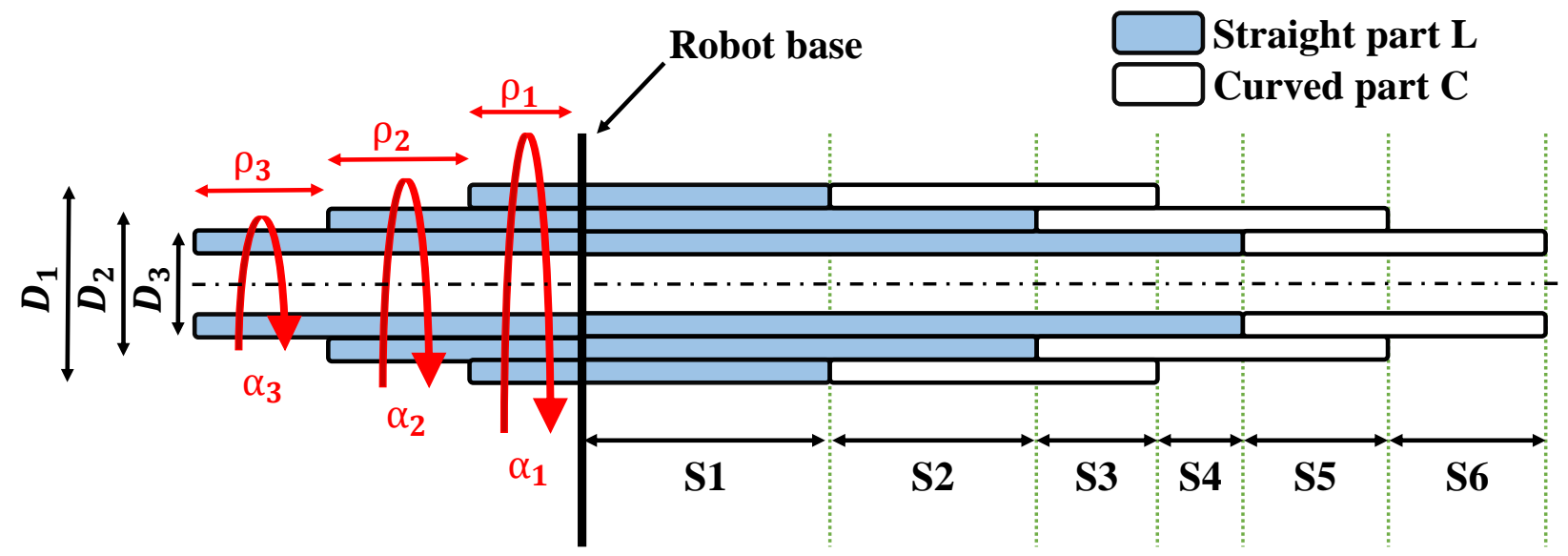

Figure 6: Definition of the $m=6$ sections when $n=3$ tubes are in interaction. A new section must be considered when the shape $\left(L_{i}\right.$ or $\left.C_{i}\right)$ or the diameter $D_{i}$ of a tube is different. Each tube can be translated by $\rho_{i}$ and rotated about its central axis by $\alpha_{i}$.

The model studied in this paper assumes three perfectly curved $\left(\kappa_{i} \neq 0\right)$ concentric tubes $i$ (with $i \in\{1 . . n\}, n=3$ ), the three links $j$ (with $j \in\{1 . . m\}, m=3$ ) can be described in Figure 7 .

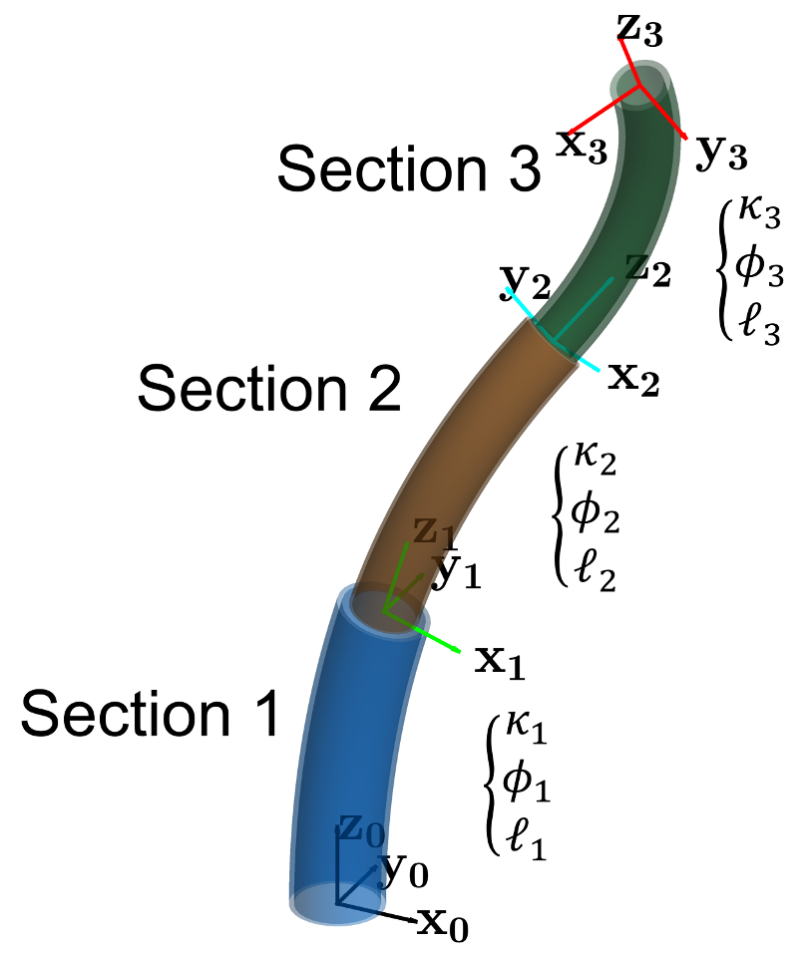

Figure 7: Schematic description of the link distribution and parameters in the presence of three totally curved concentric tubes (outer tube in blue, middle tube in brown, and inner tube in green). The general fixed frame $\left\{x_{0}, y_{0}, z_{0}\right\}$ is attached to the outer tube basis. The $z$-axis denotes the robot principal axis. A configuration frame is related to each link distal vertex (first in light green, second in dark blue, and third in red). 
The resultant shape of the robot stems from the balancing constant moments applied to the overlapping tubes, based on the Euler-Bernoulli beam theory. This assumption was theoretically proposed and experimentally validated in several works led by Sears and Dupont (2006, 2007); Webster III et al. (2006b, 2008, 2009); Rucker et al. (2010b), among others. The piece-wise constant curvature was validated by Gravagne et al. (2003) when a constant moment is applied along a beam. These results were also validated by a finite element model by Baek et al. (2015). Thus, depending on the number of the $n$ component tubes, the shape of the $j^{\text {th }}$ link results from their interaction. This interaction is illustrated by the resultant curvature in equation 1 and the equilibrium plane angle in equation 2. The inserted angle $\alpha_{i}$ of the $i^{\text {th }}$ tube about the $j^{\text {th }}$ link frame $z$-axis and the intrinsic curvature $\kappa_{i, j}$ of the portion of the tube involved in the $j^{\text {th }}$ link are taken into account as follows:

$$
\kappa_{j}=\sqrt{\kappa_{x_{j}}^{2}+\kappa_{y_{j}}^{2}} \quad \text { with } \quad \kappa_{x_{j}}=\frac{\sum_{i=1}^{n} E_{i} I_{i} \kappa_{i, j} \cos \alpha_{i}}{\sum_{i=1}^{n} E_{i} I_{i}}, \kappa_{y_{j}}=\frac{\sum_{i=1}^{n} E_{i} I_{i} \kappa_{i, j} \sin \alpha_{i}}{\sum_{i=1}^{n} E_{i} I_{i}}
$$

where $\kappa_{x}$ and $\kappa_{y}$ are the decomposition of the main curvature along the $x$ and $y$ axes respectively, $E_{i}$ is the elastic modulus, $I_{i}$ is the cross sectional moment of inertia, and:

$$
\phi_{j}=\arctan 2\left(\kappa_{y_{j}}, \kappa_{x_{j}}\right)
$$

Considering the initial pose of the robot where all the tubes are withdrawn, the link lengths are such that $\ell_{j}=0, \forall j=\{1 . .3\}$ at $t=0$. Whenever $t>0$, the tubes are deployed so that the link lengths are written as $\ell_{1}=\rho_{1}, \ell_{2}=\rho_{2}-\rho_{1}$, and $\ell_{3}=\rho_{3}-\rho_{2}$ such that $\rho_{3} \geq \rho_{2} \geq \rho_{1}$.

Three spaces were specified by Jones and Walker (2005) and later by Webster III and Jones (2010). The Cartesian task space $S E(3)$ and the actuator space $\mathbf{q} \in \mathbb{R}^{2 n}$ are linked by an intermediary space: the configuration space $\chi \in \mathbb{R}^{2 m}$ of the aforementioned arc parameters, where $\chi=\left[\begin{array}{lll}\chi_{1} & \ldots & \chi_{m}\end{array}\right]^{T}$ and $\chi_{j}=\left[\begin{array}{ll}\kappa_{j} & \phi_{j} \ell_{j}\end{array}\right]^{T}$ for $j \in\{1 . . m\}$. Two space transformations are thus defined:

1. The specific mapping (SM) from the actuator space to the configuration space (actuator dynamics) denoted $\chi=S M(\mathbf{q})$. This mapping totally depends on the actuation of the tubes. Hence, it can be adapted to the technological solution,.

2. The independent mapping from the configuration space to the task space based on forward kinematic modeling $(F K M)$ denoted ${ }^{0} T_{m}=F K M(\chi)$. This mapping is the same for all the concentric tube configurations, satisfying the assumption of constant curvature links and can be generically modeled.

As mentioned above, forward kinematics can be accomplished in a variety of ways. Despite the diversity of formalisms, frame choices and symbols employed, we obtain the same result for the transformation matrix. Indeed, the transformation ${ }^{j-1} \mathbf{T}_{j}$ from link $j-1$ to link $j$ decomposes into a rotation of center $\mathbf{r}_{j}=\left[1 / \kappa_{j}, 0,0\right]^{T}$ about the $y$ axis by $\theta_{j}$ and a rotation about the $z$ axis by $\phi_{j}:$

$$
{ }^{j-1} \mathbf{T}_{j}=\left[\begin{array}{cc}
\mathbf{R}_{z}\left(\phi_{j}\right) & 0 \\
0 & 1
\end{array}\right]\left[\begin{array}{cc}
\mathbf{R}_{y}\left(\theta_{j}\right) & \mathbf{p}_{j} \\
0 & 1
\end{array}\right]
$$

where $\theta_{j}=\kappa_{j} \ell_{j}$ and $\mathbf{p}_{j}=\left[r_{j}\left(1-\cos \theta_{j}\right), 0, r_{j} \sin \theta_{j}\right]^{T}$. 


\subsubsection{Independent kinematic Jacobian matrix}

The independent kinematic Jacobian computation relies substantially on the forward kinematic derivation. Consequently, as derived by Webster III and Jones (2010), the velocity of the $j^{\text {th }}$ link is defined according to the $j-1^{\text {th }}$ one. Thus, $\mathbf{V}_{j}$ is defined regardless of the configurations of the other sections (bending plane, curvature, or length).

$$
\mathbf{V}_{j}=\left[\begin{array}{ccc}
\cos \Delta \phi_{j}\left(\cos \left(\kappa_{j} \ell_{j}\right)-1\right) / \kappa_{j}^{2} & 0 & 0 \\
\sin \Delta \phi_{j}\left(\cos \left(\kappa_{j} \ell_{j}\right)-1\right) / \kappa_{j}^{2} & 0 & 0 \\
-\left(\sin \left(\kappa_{j} \ell_{j}\right)-\kappa_{j} \ell_{j}\right) / \kappa_{j}^{2} & 0 & 1 \\
-\ell_{j} \sin \Delta \phi_{j} & 0 & -\kappa_{j} \sin \Delta \phi_{j} \\
\ell_{j} \cos \Delta \phi_{j} & 0 & \kappa_{j} \cos \Delta \phi_{j} \\
0 & 1 & 0
\end{array}\right]\left[\begin{array}{c}
\dot{\kappa}_{j} \\
\Delta \dot{\phi}_{j} \\
\dot{\ell}_{j}
\end{array}\right]=\mathbf{J}_{\text {indep }_{j}}\left[\begin{array}{c}
\dot{\kappa}_{j} \\
\Delta \dot{\phi}_{j} \\
\dot{\ell}_{j}
\end{array}\right]
$$

where $\Delta \phi_{j}=\phi_{j}-\phi_{j-1}$ and $\Delta \phi_{1}=\phi_{1}$.

Using the adjoint transformation introduced by Murray et al. (1994), the full independent kinematic Jacobian can be deduced from the individual ones:

$$
\mathbf{J}_{\text {indep }}=\left[\begin{array}{lllll}
\mathbf{J}_{\text {indep } 1_{1}} & A d\left({ }^{0} \mathbf{T}_{1}\right) \mathbf{J}_{\text {indep } 2_{2}} & A d\left({ }^{0} \mathbf{T}_{2}\right) \mathbf{J}_{\text {indep }_{3}} & \ldots & A d\left({ }^{0} \mathbf{T}_{1(m-1)}\right) \mathbf{J}_{\text {indep }_{m}}
\end{array}\right]
$$

where $\mathbf{J}_{\text {indep }} \in \mathbb{R}^{6 \times 3 m}$ and ${ }^{0} \mathbf{T}_{j}={ }^{0} \mathbf{T}_{1}{ }^{1} \mathbf{T}_{2} \ldots{ }^{k-1} \mathbf{T}_{j}$ is the $j^{\text {th }}$ transformation matrix at the $j^{\text {th }}$ link according to the robot base, for $k \in\{1 . . j\} . A d(\mathbf{T})=\left[\begin{array}{cc}\mathbf{R} & \mathbf{R}[\mathbf{t}]_{\times} \\ 0 & \mathbf{R}\end{array}\right]$ where $\mathbf{R}$ and $\mathbf{t}$ are the rotation and translation components of $\mathbf{T}$ respectively, and $[\mathbf{t}]_{\times}$the skew-symmetric matrix associated to the vector cross-product by $\mathbf{t}$. This leads the robot end-effector velocity expression:

$$
\mathbf{V}=\mathbf{J}_{\text {indep }} \dot{\chi}
$$

Thus, determining the number of links in a configuration is a preliminary task to obtain the dimension of the full kinematic Jacobian matrix. In all the cases studied in the following subsections, we assume a continuum robot with three concentric tubes that are totally curved. Thus, the structure can be decomposed into three links as previously described in Figure 7. Consequently, we obtain $\mathbf{J}_{\text {indep }} \in \mathbb{R}^{6 \times 9}$. The following models are developed according to this assumption ( $n=3$ and $m=3$ ).

\subsection{Forward kinematic models and specific kinematic Jacobian matrices}

As the specific mapping depends essentially on the actuator technology, structure, and distribution along the continuum robot, geometric and kinematic models will be different from each other. Nevertheless, for the clarity of the paper, we use hereby the same formalisms and derivations in order to obtain results as homogeneous as possible. The derivatives of the curvature and equilibrium angle have the same structure in all cases (constant curvature model, variable curvature model, double direction variable curvature model). Differentiating (1) and (2) with respect to $\kappa_{x}$ and $\kappa_{y}$ yields:

$$
\dot{\kappa}_{j}=\frac{1}{\sqrt{\kappa_{x_{j}}^{2}+\kappa_{y_{j}}^{2}}}\left[\begin{array}{ll}
\kappa_{x_{j}} & \kappa_{y_{j}}
\end{array}\right]\left[\begin{array}{l}
\dot{\kappa}_{x_{j}} \\
\dot{\kappa}_{y_{j}}
\end{array}\right]
$$


and

$$
\dot{\phi}_{j}=\frac{1}{\kappa_{x_{j}}^{2}+\kappa_{y_{j}}^{2}}\left[\begin{array}{ll}
-\kappa_{y_{j}} & \kappa_{x_{j}}
\end{array}\right]\left[\begin{array}{c}
\dot{\kappa}_{x_{j}} \\
\dot{\kappa}_{y_{j}}
\end{array}\right]
$$

where $\dot{\kappa}_{x_{j}}$ and $\dot{\kappa}_{y_{j}}$ are respectively the derivatives of $\kappa_{x_{j}}$ and $\kappa_{y_{j}}$ with respect to the actuators used in each case. This yields the curvature and the equilibrium angle derivatives of the $j^{\text {th }}$ link:

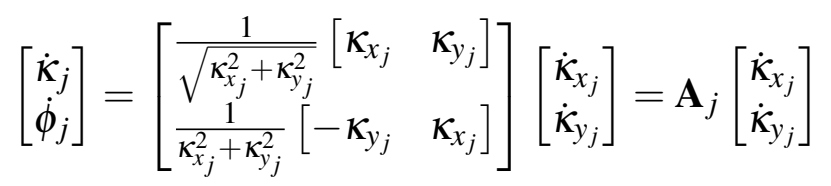

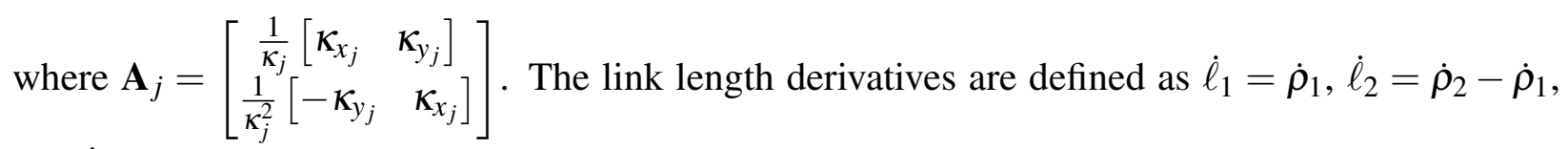
and $\dot{\ell}_{3}=\dot{\rho}_{3}-\dot{\rho}_{2}$.

Although the first configuration is studied in the literature, the explicit development of a specific Jacobian matrix is performed hereby for the first time to the best of our knowledge. We choose a development formalism in accordance with the two novel configuration models in order to perform a coherent comparison subsequently.

\subsubsection{Constant curvature model (CCM)}

The robot configuration of the constant curvature model discussed hereby was previously derived in the literature (e.g. Webster III et al. (2006b); Sears and Dupont (2006)).

The actuator space is $\left\{\mathbf{q}_{i}=\alpha_{i}, \rho_{i} \mid i \in\{1 . . n\}\right\}$ where $\alpha_{i}$ and $\rho_{i}$ are the insertion angle and length of the $i^{t h}$ tube respectively. In order to permit a generic modeling, the formalism used hereby is the matrix notation. In the following, the curvature components of each link will be differentiated according to the actuator derivatives:

$$
\left[\begin{array}{c}
\dot{\kappa}_{x_{j}} \\
\dot{\kappa}_{y_{j}}
\end{array}\right]=\mathbf{B}_{j}\left[\begin{array}{lll}
\dot{\alpha}_{j} & \ldots & \dot{\alpha}_{m}
\end{array}\right]^{T}
$$

Explicit examples of the following developments are detailed in the Appendix ( $c f$. equation 40) Differentiating the curvature components of the third link leads to:

$$
\left[\begin{array}{c}
\dot{\kappa}_{x_{3}} \\
\dot{\kappa}_{y_{3}}
\end{array}\right]=\left[\begin{array}{c}
-\kappa_{i, 3} \sin \alpha_{3} \\
\kappa_{i, 3} \cos \alpha_{3}
\end{array}\right] \dot{\alpha}_{3}=\mathbf{B}_{3} \dot{\alpha}_{3}
$$

resulting into the third link curvature and equilibrium angle derivatives:

$$
\left[\begin{array}{c}
\dot{\kappa}_{3} \\
\dot{\phi}_{3}
\end{array}\right]=\mathbf{A}_{3} \mathbf{B}_{3} \dot{\alpha}_{3}
$$

Similarly, for the second link, the curvature component derivatives are:

$$
\left[\begin{array}{l}
\dot{\kappa}_{x_{2}} \\
\dot{\kappa}_{y_{2}}
\end{array}\right]=\mathbf{B}_{2}\left[\begin{array}{l}
\dot{\alpha}_{2} \\
\dot{\alpha}_{3}
\end{array}\right]
$$


with $\mathbf{B}_{2}=\frac{1}{E_{2} I_{2}+E_{3} I_{3}}\left[\begin{array}{cc}-E_{2} I_{2} \kappa_{i, 2} \sin \alpha_{2} & -E_{3} I_{3} \kappa_{i, 3} \sin \alpha_{3} \\ E_{2} I_{2} \kappa_{i, 2} \cos \alpha_{2} & E_{3} I_{3} \kappa_{i, 3} \cos \alpha_{3}\end{array}\right]$, yielding the second link curvature and equilibrium angle derivatives:

$$
\left[\begin{array}{c}
\dot{\kappa}_{2} \\
\dot{\phi}_{2}
\end{array}\right]=\mathbf{A}_{2} \mathbf{B}_{2}\left[\begin{array}{c}
\dot{\alpha}_{2} \\
\dot{\alpha}_{3}
\end{array}\right]
$$

For the first link, the curvature component derivatives can be written:

$$
\left[\begin{array}{c}
\dot{\kappa}_{x_{1}} \\
\dot{\kappa}_{y_{1}}
\end{array}\right]=\mathbf{B}_{1}\left[\begin{array}{lll}
\dot{\alpha}_{1} & \dot{\alpha}_{2} & \dot{\alpha}_{3}
\end{array}\right]^{T}
$$

with $\mathbf{B}_{1}=\frac{1}{E_{1} I_{1}+E_{2} I_{2}+E_{3} I_{3}}\left[\begin{array}{ccc}-E_{1} I_{1} \kappa_{i, 1} \sin \alpha_{1} & -E_{2} I_{2} \kappa_{i, 2} \sin \alpha_{2} & -E_{3} I_{3} \kappa_{i, 3} \sin \alpha_{3} \\ E_{1} I_{1} \kappa_{i, 1} \cos \alpha_{1} & E_{2} I_{2} \kappa_{i, 2} \cos \alpha_{2} & E_{3} I_{3} \kappa_{i, 3} \cos \alpha_{3}\end{array}\right]$, providing the first link curvature and equilibrium angle derivatives:

$$
\left[\begin{array}{c}
\dot{\kappa}_{1} \\
\dot{\phi}_{1}
\end{array}\right]=\mathbf{A}_{1} \mathbf{B}_{1}\left[\begin{array}{lll}
\dot{\alpha}_{1} & \dot{\alpha}_{2} & \dot{\alpha}_{3}
\end{array}\right]^{T}
$$

To summarize, after adding the link length derivatives, the final arc parameter derivatives for the constant curvature configuration is:

$$
\dot{\chi}=\left[\begin{array}{cccccc}
\multicolumn{2}{c}{\mathbf{X}_{1}} & & 0_{2 \times 3} & \\
0_{2 \times 1} & \mathbf{X}_{2} & 0_{2 \times 3} & \\
0_{2 \times 2} & \mathbf{X}_{3} & 0_{2 \times 3} & \\
0 & 0 & 0 & 1 & 0 & 0 \\
0 & 0 & 0 & -1 & 1 & 0 \\
0 & 0 & 0 & 0 & -1 & 1
\end{array}\right] \dot{\mathbf{q}}_{C C M}=\mathbf{J}_{\text {spec }}^{C C M} \dot{\mathbf{q}}_{C C M}
$$

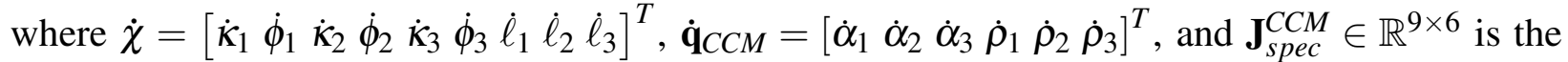
specific kinematic Jacobian for the constant curvature model (CCM), with $\mathbf{X}_{1}=\mathbf{A}_{1} \mathbf{B}_{1}, \quad \mathbf{X}_{2}=$ $\mathbf{A}_{2} \mathbf{B}_{2}$, and $\mathbf{X}_{3}=\mathbf{A}_{3} \mathbf{B}_{3}$.

\subsubsection{Variable curvature model (VCM)}

This configuration was previously developed in details by Chikhaoui et al. (2014a). A different formalism, which is coherent with the other developed models, is presented hereby. The actuator space in this case is $\left\{\mathbf{q}_{i}=v_{i}, \alpha_{i}, \rho_{i} \mid i \in\{1 . . n\}\right\} . v_{i}$ is the applied voltage to the $i^{t h}$ tube (cf. equation 18), $\alpha_{i}$ and $\rho_{i}$ are the insertion angle and length of the $i^{t h}$ tube respectively. The tube curvature, which was previously a passive variable of the system, is now accessible. One can express the inserted curvature $\kappa_{i n s, i}$ of the $i^{\text {th }}$ tube in terms of the applied voltage $v_{i}$ as follows:

$$
\kappa_{i n s, i}=C_{P P y_{i}} v_{i}
$$

where the PPy constant $C_{P P y_{i}}$, related to the $i^{t h}$ tube, is given by:

$$
C_{P P y_{i}}=\frac{32}{3 \pi} \frac{E_{p} \lambda C_{v}\left[a_{i}^{3}-\left(a_{i}+2 t_{p}\right)^{3}\right]}{E_{i} b_{i}^{4}-E_{p}\left(a_{i}+2 t_{p}\right)^{4}-E_{i} a_{i}^{4}+E_{p} a_{i}^{4}}
$$


$E_{i}$ and $E_{p}$ are the Young's moduli of the $i^{t h}$ tube and the PPy respectively, $a_{i}$ and $b_{i}$ are the outer and inner diameters of the $i^{t h}$ tube respectively, and $t_{p}$ represents the thickness of the PPy electrodes, $\lambda$ is an empirically determined strain to volumetric charge ratio, $C_{v}$ is the volumetric capacitance of the polymer, and $v_{i}$ is the applied voltage.

Similarly as for the previous model (CCM), details of an example of link parameter derivations is introduced in the Appendix ( $c f$. equation 41). Differentiation of the curvature components of the third link is carried out and gives:

$$
\left[\begin{array}{c}
\dot{\kappa}_{x_{3}} \\
\dot{\kappa}_{y_{3}}
\end{array}\right]=\mathbf{C}_{3}\left[\begin{array}{llllll}
0 & 0 & \dot{v}_{3} & 0 & 0 & \dot{\alpha}_{3}
\end{array}\right]^{T}
$$

where $\mathbf{C}_{3}=\left[\begin{array}{cccccc}0 & 0 & \cos \alpha_{3} C_{P P y_{3}} & 0 & 0 & -\kappa_{i, 3} \sin \alpha_{3} \\ 0 & 0 & \sin \alpha_{3} C_{P P y_{3}} & 0 & 0 & \kappa_{i, 3} \cos \alpha_{3}\end{array}\right]$, yielding the third link curvature and equilibrium angle derivatives:

$$
\left[\begin{array}{c}
\dot{\kappa}_{3} \\
\dot{\phi}_{3}
\end{array}\right]=\mathbf{A}_{3} \mathbf{C}_{3}\left[\begin{array}{llllll}
0 & 0 & \dot{v}_{3} & 0 & 0 & \dot{\alpha}_{3}
\end{array}\right]^{T}
$$

Similarly, derivatives of the second link curvature components can be established:

$$
\left[\begin{array}{c}
\dot{\kappa}_{x_{2}} \\
\dot{\kappa}_{y_{2}}
\end{array}\right]=\mathbf{C}_{2}\left[\begin{array}{llllll}
0 & \dot{v}_{2} & \dot{v}_{3} & 0 & \dot{\alpha}_{2} & \dot{\alpha}_{3}
\end{array}\right]^{T}
$$

where $\mathbf{C}_{2}=\frac{1}{E_{2} I_{2}+E_{3} I_{3}} \mathbf{C}_{2}^{\prime}$ with

$\mathbf{C}_{2}^{\prime}=\left[\begin{array}{llllll}0 & E_{2} I_{2} \cos \alpha_{2} C_{P P y_{2}} & E_{3} I_{3} \cos \alpha_{3} C_{P P y_{3}} & 0 & -E_{2} I_{2} \kappa_{i, 2} \sin \alpha_{2} & -E_{3} I_{3} \kappa_{i, 3} \sin \alpha_{3} \\ 0 & E_{2} I_{2} \sin \alpha_{2} C_{P P y_{2}} & E_{3} I_{3} \sin \alpha_{3} C_{P P y_{3}} & 0 & E_{2} I_{2} \kappa_{i, 2} \cos \alpha_{2} & E_{3} I_{3} \kappa_{i, 3} \cos \alpha_{3}\end{array}\right]$, giving the second link curvature and equilibrium angle derivatives:

$$
\left[\begin{array}{c}
\dot{\kappa}_{2} \\
\dot{\phi}_{2}
\end{array}\right]=\mathbf{A}_{2} \mathbf{C}_{2}\left[\begin{array}{llllll}
0 & \dot{v}_{2} & \dot{v}_{3} & 0 & \dot{\alpha}_{2} & \dot{\alpha}_{3}
\end{array}\right]^{T}
$$

Finally, for the first link, the curvature component derivatives are:

$$
\left[\begin{array}{c}
\dot{\kappa}_{x_{1}} \\
\dot{\kappa}_{y_{1}}
\end{array}\right]=\mathbf{C}_{1}\left[\begin{array}{llllll}
\dot{v}_{1} & \dot{v}_{2} & \dot{v}_{3} & \dot{\alpha}_{1} & \dot{\alpha}_{2} & \dot{\alpha}_{3}
\end{array}\right]^{T}
$$

where $\mathbf{C}_{1}=\frac{1}{E_{1} I_{1}+E_{2} I_{2}+E_{3} I_{3}} \mathbf{C}_{1}^{\prime}$ with

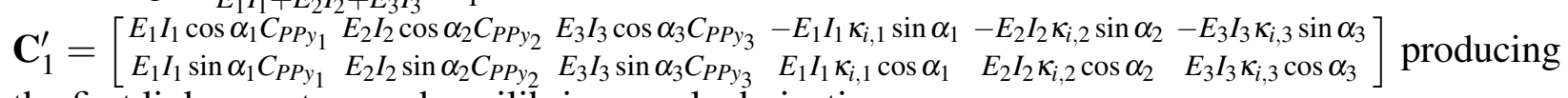
the first link curvature and equilibrium angle derivatives:

$$
\left[\begin{array}{c}
\dot{K}_{1} \\
\dot{\phi}_{1}
\end{array}\right]=\mathbf{A}_{1} \mathbf{C}_{1}\left[\begin{array}{llllll}
\dot{v}_{1} & \dot{v}_{2} & \dot{v}_{3} & \dot{\alpha}_{1} & \dot{\alpha}_{2} & \dot{\alpha}_{3}
\end{array}\right]^{T}
$$


To summarize, the final arc parameter derivatives for this case, when adding the link length derivative, produces:

$$
\dot{\chi}=\left[\begin{array}{cccc}
\mathbf{Y}_{1} & 0_{2 \times 3} & \\
\mathbf{Y}_{2} & 0_{2 \times 3} & \\
\mathbf{Y}_{3} & 0_{2 \times 3} & \\
0_{1 \times 6} & 1 & 0 & 0 \\
0_{1 \times 6} & -1 & 1 & 0 \\
0_{1 \times 6} & 0 & -1 & 1
\end{array}\right] \dot{\mathbf{q}}_{V C M}=\mathbf{J}_{\text {spec }}^{V C M} \dot{\mathbf{q}}_{V C M}
$$

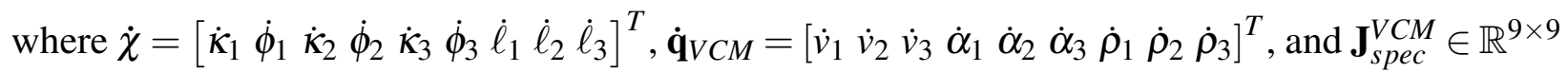
is the specific kinematic Jacobian matrix for the variable curvature model (VCM), with $\mathbf{Y}_{1}=$ $\mathbf{A}_{1} \mathbf{C}_{1}, \quad \mathbf{Y}_{2}=\mathbf{A}_{2} \mathbf{C}_{2}$, and $\mathbf{Y}_{3}=\mathbf{A}_{3} \mathbf{C}_{3}$.

\subsubsection{Double direction variable curvature model (DDVCM)}

As a further development of the configuration modeled in the previous subsection, controlling the curvature of the tubes in a continuum robot may enlarge the actuator space. The advantages of such improvements will be discussed in the next section. This configuration aims to offer two orthogonal bending directions for each tube. The electrodes coated around the tubes will be shaped into two couples in order to obtain one couple of antagonistic electrodes for each bending direction (c.f. Figure 3b). We suggest that the effect will be directly noticeable on the two components of the resultant curvature: $\kappa_{x_{j_{i n s}}}=C_{P P y_{j}} v_{x, j}$ and $\kappa_{y_{j_{i n s}}}=C_{P P y_{j}} v_{y, j}$. Noting that $v_{x, j}$ and $v_{y, j}$ are the voltages applied to the first and second electrode couples of the $i^{t h}$ tube, creating bending according to $x$ and $y$ axes, respectively, and $C_{P P y_{j}}$ as defined in equation 19. It denotes that the intrinsic curvature of each tube is now accessible, not only in its resultant value as demonstrated in the previous subsection, but also on its own $x$ and $y$ axis curvature components.

In this case, we define the suitable specific mapping with the new actuator space $\mathbf{q}_{i}$ for every tube with $\left\{\mathbf{q}_{i}=v_{x, i}, v_{y, i}, \rho_{i} \mid i \in\{1 . . n\}\right\} . v_{x, i}$ and, $v_{y, i}$ are the applied voltages as defined previously, and $\rho_{i}$ is the insertion length of the $i^{t h}$ tube. An example of detailed computation of the derivations is given in the Appendix ( $c f$. equation 42). The curvature components for the third link can be written as follows:

$$
\kappa_{x_{\text {ins }}}=C_{P P y_{3}} v_{x, 3} \text { and } \kappa_{y_{3 i n s}}=C_{P P y_{3}} v_{y, 3}
$$

Similarly, for the second link curvature components, we obtain:

$$
\kappa_{x_{2}}=\frac{E_{2} I_{2} \kappa_{2_{\text {ins }}} \cos \phi_{2_{\text {ins }}}+E_{3} I_{3} \kappa_{3 \text { ins }} \cos \phi_{3_{\text {ins }}}}{E_{2} I_{2}+E_{3} I_{3}} \text { and } \kappa_{y_{2}}=\frac{E_{2} I_{2} \kappa_{2_{\text {ins }}} \sin \phi_{2_{\text {ins }}}+E_{3} I_{3} \kappa_{3 \text { ins }} \sin \phi_{3_{\text {ins }}}}{E_{2} I_{2}+E_{3} I_{3}}
$$

Finally, for the first link, the curvature components are:

$$
\begin{aligned}
\kappa_{x_{1}} & =\frac{E_{1} I_{1} \kappa_{1_{\text {ins }}} \cos \phi_{1_{\text {ins }}}+E_{2} I_{2} \kappa_{2_{\text {ins }}} \cos \phi_{2_{\text {ins }}}+E_{3} I_{3} \kappa_{3_{\text {ins }}} \cos \phi_{3_{\text {ins }}}}{E_{1} I_{1}+E_{2} I_{2}+E_{3} I_{3}} \\
\text { and } \kappa_{y_{2}} & =\frac{E_{1} I_{1} \kappa_{1_{\text {ins }}} \sin \phi_{1_{\text {ins }}}+E_{2} I_{2} \kappa_{2_{\text {ins }}} \sin \phi_{2_{\text {ins }}}+E_{3} I_{3} \kappa_{3_{\text {ins }}} \sin \phi_{3_{\text {ins }}}}{E_{1} I_{1}+E_{2} I_{2}+E_{3} I_{3}}
\end{aligned}
$$


Let $\mathbf{d}_{j_{\kappa}}$ and $\mathbf{d}_{j_{\phi}}$ denote respectively: $\mathbf{d}_{j_{\kappa}}=\frac{C_{P P y_{j}}}{\sqrt{\kappa_{x_{j}}^{2}+\kappa_{y_{j}}^{2}}}\left[\begin{array}{ll}\kappa_{x_{j}} & \kappa_{y_{j}}\end{array}\right]$ and $\mathbf{d}_{j_{\phi}}=\frac{C_{P P y_{j}}}{\kappa_{x_{j}}^{2}+\kappa_{y_{j}}^{2}}\left[\begin{array}{ll}-\kappa_{y_{j}} & \kappa_{x_{j}}\end{array}\right]$, for $j \in\{1 . .3\}$. Similarly, differentiating equations 7 and 8 , taking into account equation 27 yields, for the third link:

$$
\left[\begin{array}{l}
\dot{\kappa}_{3} \\
\dot{\phi}_{3}
\end{array}\right]=\mathbf{A}_{3}\left[\begin{array}{c}
\dot{\kappa}_{x, 3} \\
\dot{\kappa}_{y, 3}
\end{array}\right]
$$

while

$$
\left[\begin{array}{c}
\dot{\kappa}_{x, 3} \\
\dot{\kappa}_{y, 3}
\end{array}\right]=\mathbf{D}_{3}\left[\begin{array}{c}
\dot{v}_{x, 3} \\
\dot{v}_{y, 3}
\end{array}\right]
$$

where $\mathbf{D}_{3}=\left[\begin{array}{cc}\cos \phi_{3} & -\kappa_{3_{\text {ins }}} \sin \phi_{3} \\ \sin \phi_{3} & \kappa_{3_{\text {ins }}} \cos \phi_{3}\end{array}\right]\left[\begin{array}{l}\mathbf{d}_{3_{\kappa}} \\ \mathbf{d}_{3_{\phi}}\end{array}\right]$.

The second link curvature and equilibrium angle can be written as follows: yielding the second link curvature and equilibrium angle derivatives:

$$
\left[\begin{array}{c}
\dot{\kappa}_{2} \\
\dot{\phi}_{2}
\end{array}\right]=\mathbf{A}_{2} \mathbf{D}_{2}\left[\begin{array}{llll}
\dot{v}_{x, 2} & \dot{v}_{y, 2} & \dot{v}_{x, 3} & \dot{v}_{y, 3}
\end{array}\right]^{T}
$$

where $\mathbf{D}_{2}=\frac{1}{E_{2} I_{2}+E_{3} I_{3}}\left[\begin{array}{llll}E_{2} I_{2} \cos \phi_{2} & -E_{2} I_{2} \kappa_{2_{\text {ins }}} & E_{3} I_{3} \cos \phi_{3} & -E_{3} I_{3} \kappa_{3_{\text {ins }}} \\ E_{2} I_{2} \sin \phi_{2} & E_{2} I_{2} \cos \phi_{2} & E_{3} I_{3} \sin \phi_{3} & E_{3} I_{3} \cos \phi_{3}\end{array}\right]\left[\begin{array}{cc}\mathbf{d}_{2_{\kappa}} & 0_{1 \times 2} \\ \mathbf{d}_{2_{\phi}} & 0_{1 \times 2} \\ 0_{1 \times 2} & \mathbf{d}_{3_{\kappa}} \\ 0_{1 \times 2} & \mathbf{d}_{3_{\phi}}\end{array}\right]$.

Similarly, the inserted curvature derivatives of the tubes interacting in the first link:

$$
\left[\begin{array}{l}
\dot{\kappa}_{1_{\text {ins }}} \\
\dot{\kappa}_{2_{\text {ins }}} \\
\dot{\kappa}_{3_{\text {ins }}}
\end{array}\right]=\left[\begin{array}{ccc}
\mathbf{d}_{1_{\kappa}} & 0_{1 \times 2} & 0_{1 \times 2} \\
0_{1 \times 2} & \mathbf{d}_{2_{\kappa}} & 0_{1 \times 2} \\
0_{1 \times 2} & 0_{1 \times 2} & \mathbf{d}_{1_{\kappa}}
\end{array}\right]\left[\begin{array}{llllll}
\dot{v}_{x, 1} & \dot{v}_{y, 1} & \dot{v}_{x, 2} & \dot{v}_{y, 2} & \dot{v}_{x, 3} & \dot{v}_{y, 3}
\end{array}\right]^{T}
$$

yielding the first link curvature and equilibrium angle derivatives:

$$
\left[\begin{array}{c}
\dot{\kappa}_{1} \\
\dot{\phi}_{1}
\end{array}\right]=\mathbf{A}_{1} \mathbf{D}_{1}\left[\begin{array}{llllll}
\dot{v}_{x, 1} & \dot{v}_{y, 1} & \dot{v}_{x, 2} & \dot{v}_{y, 2} & \dot{v}_{x, 3} & \dot{v}_{y, 3}
\end{array}\right]^{T}
$$

where

$$
\begin{array}{cl}
\mathbf{D}_{1}=\frac{1}{E_{1} I_{1}+E_{2} I_{2}+E_{3} I_{3}} & {\left[\begin{array}{llllll}
E_{1} I_{1} \cos \phi_{1} & -E_{1} I_{1} \kappa_{1_{\text {ins }}} & E_{2} I_{2} \cos \phi_{2} & -E_{2} I_{2} \kappa_{2} \text { ins } & E_{3} I_{3} \cos \phi_{3} & -E_{3} I_{3} \kappa_{3_{\text {ins }}} \\
E_{1} I_{1} \sin \phi_{1} & E_{1} I_{1} \cos \phi_{1} & E_{2} I_{2} \sin \phi_{2} & E_{2} I_{2} \cos \phi_{2} & E_{3} I_{3} \sin \phi_{3} & E_{3} I_{3} \cos \phi_{3}
\end{array}\right]} \\
& {\left[\begin{array}{cccc}
\mathbf{d}_{\mathbf{1}_{\kappa}} & 0_{1 \times 2} & 0_{1 \times 2} \\
\mathbf{d}_{1_{\phi}} & 0_{1 \times 2} & 0_{1 \times 2} \\
0_{1 \times 2} & \mathbf{d}_{2 \kappa} & 0_{1 \times 2} \\
0_{1 \times 2} & \mathbf{d}_{2 \phi} & 0_{1 \times 2} \\
0_{1 \times 2} & 0_{1 \times 2} & \mathbf{d}_{3_{\kappa}} \\
0_{1 \times 2} & 0_{1 \times 2} & \mathbf{d}_{3_{\phi}}
\end{array}\right] .}
\end{array}
$$


To summarize, the arc parameter derivatives for the double direction variable curvature case can be expressed:

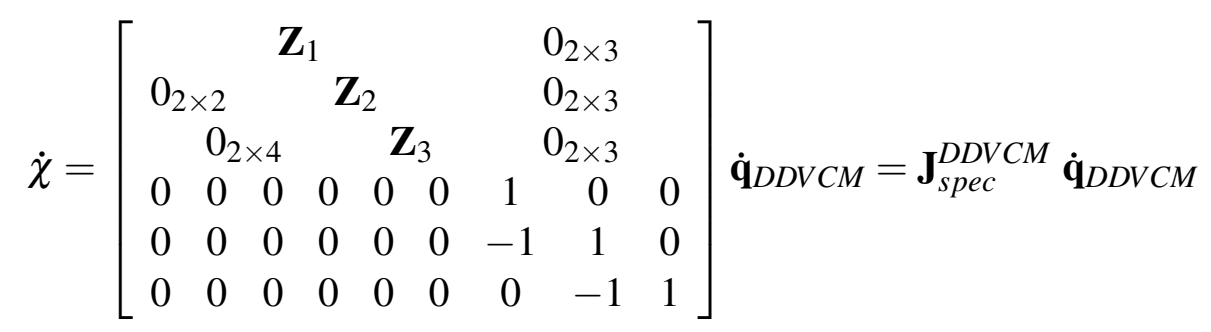

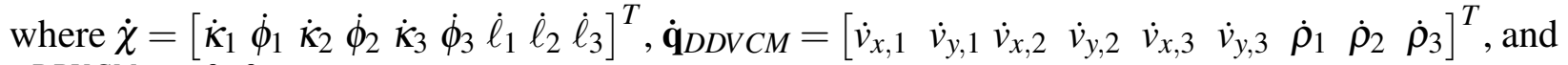
$\mathbf{J}_{\text {spec }}^{D D V C M} \in \mathbb{R}^{9 \times 9}$ is the specific kinematic Jacobian for the double direction variable curvature model (DDVCM), where $\mathbf{Z}_{1}=\mathbf{A}_{1} \mathbf{D}_{1} \in \mathbb{R}^{2 \times 6}, \quad \mathbf{Z}_{2}=\mathbf{A}_{2} \mathbf{D}_{2} \in \mathbb{R}^{2 \times 4}$, and $\mathbf{Z}_{3}=\mathbf{A}_{3} \mathbf{D}_{3} \in \mathbb{R}^{2 \times 2}$.

\subsection{Interim conclusions on modeling}

Observing the specific kinematic Jacobian matrices obtained in every case, the $\mathbf{J}_{\text {spec }}^{C C M}$ is a $6 \times 9$ matrix, in contrast with $\mathbf{J}_{\text {spec }}^{V C M}$ and $\mathbf{J}_{\text {spec }}^{D D V M}$ that are $9 \times 9$ square matrices. Thus, the specific kinematic Jacobian matrix for the standard concentric tubes can never have a full rank (yielding a non-holonomic constraint) where the arc parameters (9) are controlled with less actuators (6). Whereas, the kinematic Jacobian matrices of both proposed models are of full size. This means that each link of the robot is holonomic and so is to the entire system, regarding the configuration space parameters. In contrast with the CCM, all the velocity directions for each link of VCM and DDVCM are available. This is potentially useful when performing a linear sweeping movement for instance. The sweeping direction that is prevented in a CCM configuration can be achieved by the new holonomic manipulators, as will be shown further.

Furthermore, when comparing the two developed models VCM and DDVCM, as its kinematic Jacobian matrix is full $\left(\mathbf{J}_{\text {spec }}^{D D V C M} \in \mathbb{R}^{9 \times 9}\right)$, all the configuration space is accessible for the latter without controlling the insertion angle $\alpha_{i}$. Consequently, one can get rid of the rotation actuators (controlling $\alpha_{i}$ ) when adding the double direction curvature control (cf. Figure 4). This is potentially helpful for a quick Operating Room (OR) introduction due to the small size of the actuators.

\section{Numerical results}

In order to validate the aforementioned models developed herein, let us carry out numerical simulations concerning the workspace in terms of volume covered as well as pose occurrences that might ensue. The specifications of the robot used in numerical simulations are detailed in Table 2. 


\begin{tabular}{l||c|c|c}
\multicolumn{4}{c}{ Table 2: Tube specifications } \\
Tube number & 1 (outer) & 2 (middle) & 3 (inner) \\
\hline Outer diameter $(\mathrm{mm})$ & 5 & 1.8 & 0.6 \\
Inner diameter $(\mathrm{mm})$ & 2 & 1.5 & 0.4 \\
Total length $(\mathrm{mm})$ & 15 & 30 & 45 \\
Curvature $\left(\mathrm{m}^{-1}\right)$ & 20 & 50 & 80 \\
Cross-sectional moment of inertia $\left(\mathrm{m}^{4}\right)$ & $\simeq 2.9910^{-11}$ & $\simeq 2.6710^{-13}$ & $\simeq 5.1110^{-15}$ \\
Shape & \multicolumn{3}{|c}{ Totally curved } \\
Young Modulus $(\mathrm{GPa})$ & \multicolumn{3}{|c}{0.7}
\end{tabular}

Numerical simulations concern also the kinematic performances of the system. This is described by analyzing the different full kinematic Jacobian matrices in terms of characteristic parameters. For each model, the robot kinematic Jacobian is defined as follows:

$$
\mathbf{J}_{\text {Robot }}^{C C M}=\mathbf{J}_{\text {indep }} \mathbf{J}_{\text {spec }}^{C C M}, \quad \mathbf{J}_{\text {Robot }}^{V C M}=\mathbf{J}_{\text {indep }} \mathbf{J}_{\text {spec }}^{V C M}, \quad \text { and } \mathbf{J}_{\text {Robot }}^{D D V C M}=\mathbf{J}_{\text {indep }} \mathbf{J}_{\text {spec }}^{D D V C M}
$$

Note that $\mathbf{J}_{\text {indep }}$ is rearranged in order to conform with the arc parameter derivatives $\dot{\chi}$ used in equations 17,26 , and 35 . Doing so, we enable singularity detection using singular value decomposition. Finally, a further step of analysis examines the performance indices to provide us with a quantitative meaning.

\subsection{Workspace}

\subsubsection{Covering}

In order to have homogeneous workspace point clouds, a set of 262.144 points denoted $\Gamma$ was generated. The discretization is performed on this basis for the three models analyzed. Also, the ranges of the insertion angles for CCM and VCM are the same; while the ranges of the tube curvatures are the same for the three models (varying between 0 and $\pm 20 \mathrm{~m}^{-1}$ for the first tube, $\pm 50 \mathrm{~m}^{-1}$ for the second tube, and $\pm 80 \mathrm{~m}^{-1}$ for the third tube). The sampling is performed with 8 steps for the inserted lengths and angles for CCM. For VCM and DDVCM, 4 steps are used for the inserted lengths, angles, and the variable curvatures. Inside the principal loop of the inserted lengths for all the models, the secondary loop of the inserted angles is followed for CCM and VCM. For the latter, a last loop of the variable curvatures is crossed afterward, while the principal loop is performed for DDVCM and followed by the double variable curvature loop.

Figure 8 displays the CCM, VCM and DDVCM reachable workspaces. Increasing the curvatures $\left(\kappa_{1}, \kappa_{2}\right.$, and $\left.\kappa_{3}\right)$ of about $25 \%$ impacts the covered workspaces. This increase is of approximately $36 \%$ for VCM and for DDVCM compared to the previous workspaces. Projected views of the three studied workspaces are displayed on Figure 9 and the covered volumes of the workspaces are summarized in Table 3. 
a)

CCM workspace

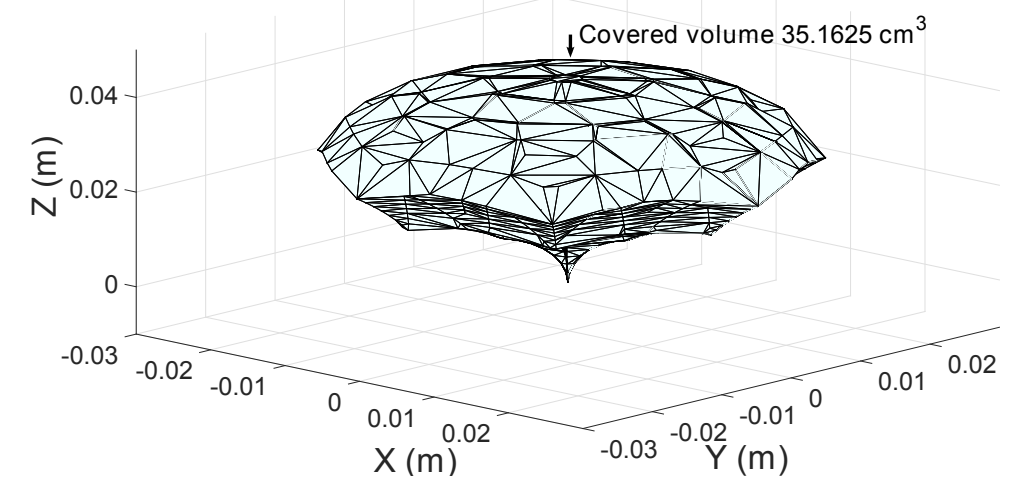

b)

VCM workspace

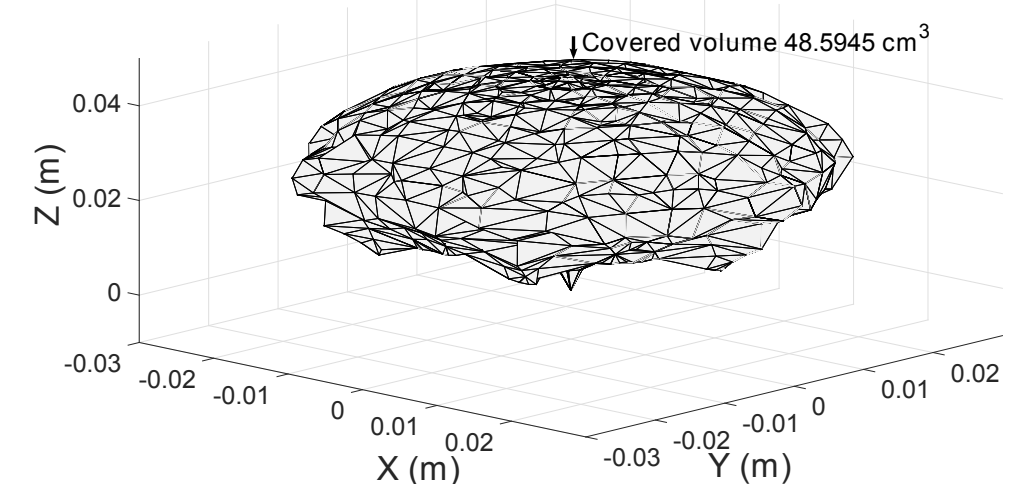

c)

\section{DDVCM workspace}

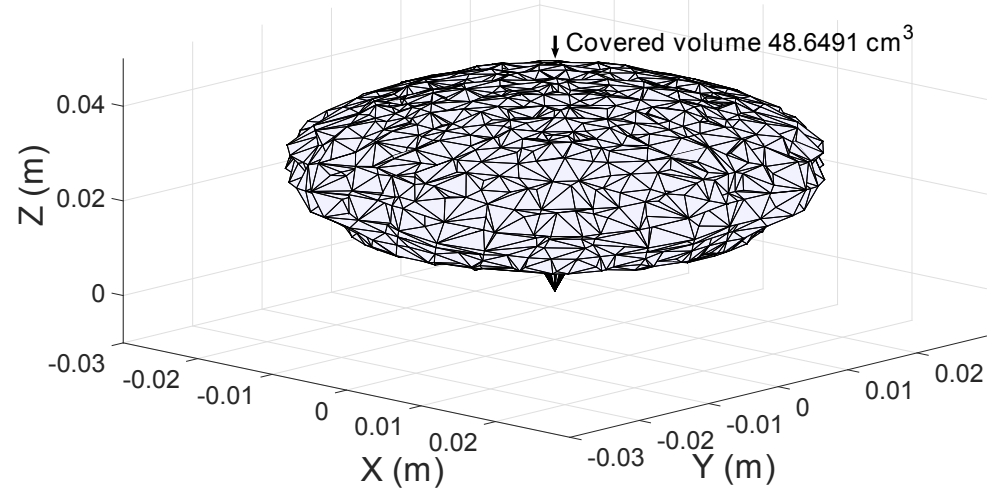

Figure 8: a) Workspace covering of CCM with tube lengths $\rho_{i} \in[0 ; 15 \mathrm{~mm}]$ and insertion angles $\theta_{i} \in[0 ; 3 \pi / 2]$. b) Workspace covering of VCM with tube lengths $\rho_{i} \in[0 ; 15 \mathrm{~mm}]$, insertion angles $\theta_{i} \in[0 ; 3 \pi / 2]$, and tube curvatures $\kappa_{1} \in\left[-25 ; 25 m^{-1}\right], \kappa_{2} \in\left[-62.5 ; 62.5 m^{-1}\right]$, and $\kappa_{3} \in\left[-100 ; 100 m^{-1}\right]$. c) Workspace covering of DDVCM with tube lengths $\rho_{i} \in[0 ; 15 \mathrm{~mm}]$ and tube curvature components $\kappa_{1_{x, y}} \in\left[-17.7 ; 17.7 \mathrm{~m}^{-1}\right], \kappa_{2_{x, y}} \in\left[-44.2 ; 44.2 \mathrm{~m}^{-1}\right]$, and $\kappa_{3_{x, y}} \in$ $\left[-70.7 ; 70.7 m^{-1}\right]$. 
a)

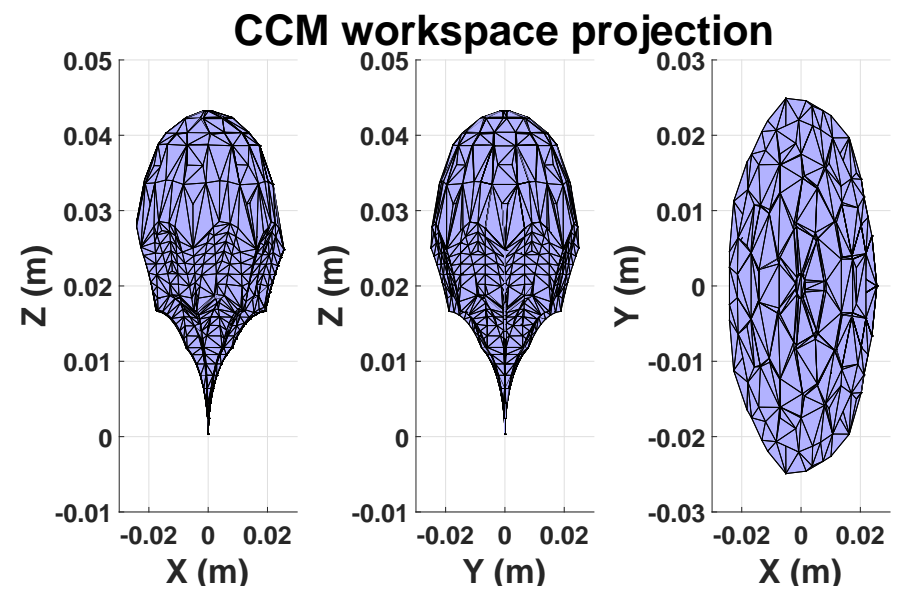

b)

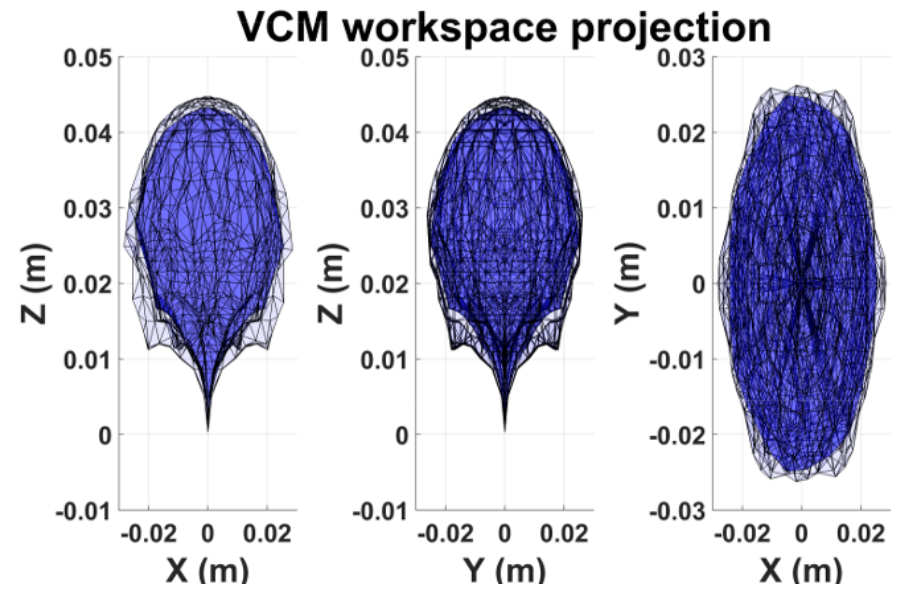

c)

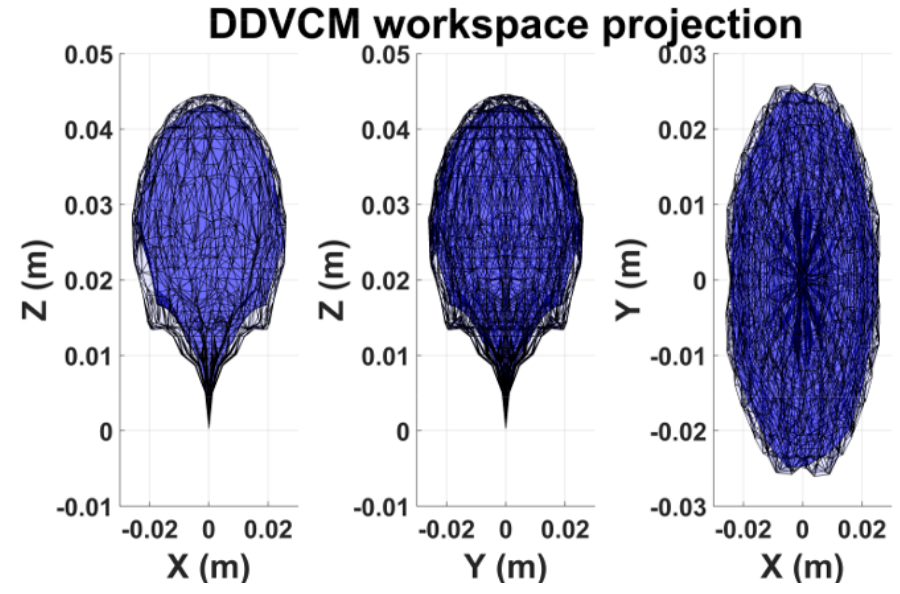

Figure 9: Workspace covering projections of Figure 8 of a) CCM, b) VCM, and c) DDVCM on the $x-z, y-z$, and $x-y$ planes respectively from left to right. The transparent plots of b) and c) are those of VCM and DDVCM respectively, and the dark plot is that of CCM. 
Table 3: Comparison of the workspace covered volume

\begin{tabular}{l||c|c|c} 
Model & Initial volume $\vartheta\left(\mathrm{cm}^{3}\right)$ & Curvature increase & Final volume $\vartheta^{\prime}\left(\mathrm{cm}^{3}\right)$ \\
\hline CCM & $\sim 35$ & not possible after tube assembly & $\sim 35$ \\
VCM & $\sim 35$ & $25 \%$ & $\sim 48$ \\
DDVCM & $\sim 35$ & $25 \%$ & $\sim 48$
\end{tabular}

\subsubsection{Pose occurrences}

In a given volume $\left(e . g .1 \mathrm{~mm}^{3}\right)$ of the workspace, a pose occurrence is defined as the cluster of the robot end-effector poses that fall into this volume with less than a given degree (e.g. $\left.10^{\circ}\right)$ difference from a central orientation. Detecting pose occurrences among all the achievable poses of the robot is important in two aspects: (i) to determine the actuation and the orientation options that an operator can have in order to reach a target point and (ii) to distinguish the different paths that the manipulator may follow while navigating into the human body. The numerical simulations are carried out for each model as follows:

a)

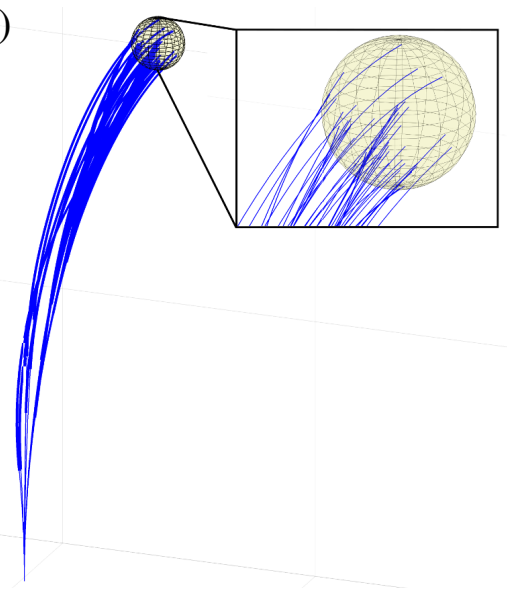

b)

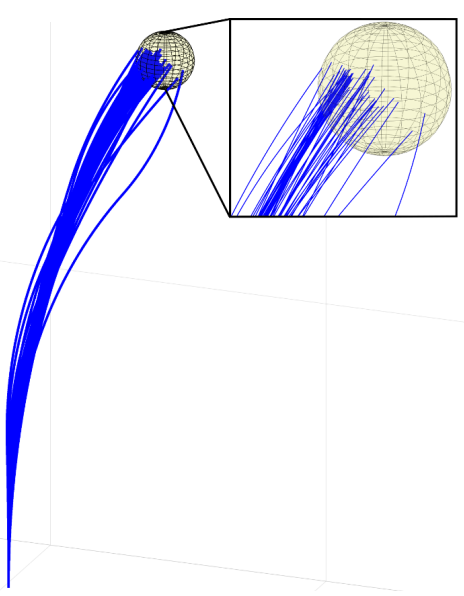

c)

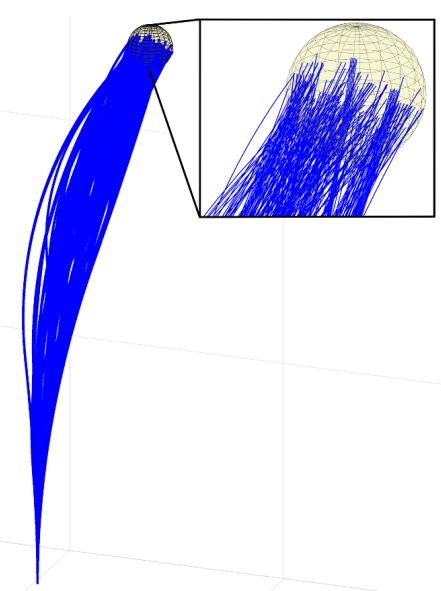

Figure 10: Pose occurrences at three random points of $L>30 \mathrm{~mm}$ (to ensure three tube extension) among the workspace subset $\Gamma_{\text {sub }}$ at $R=1 \mathrm{~mm}$ distance and $\delta=10^{\circ}$ deviation yielding a) 86 occurrences for CCM, b) 136 occurrences for VCM, and d) 229 occurrences for DDVCM. The insets are zoomed views of the end-effectors and the transparent spheres denote the random reference point neighborhood $(R=1 \mathrm{~mm})$.

- the workspace point cloud $\Gamma$ is loaded (end-effector positions and orientations) as well as the respective arc parameters $\chi$ (curvatures, equilibrium angles, and insertion lengths),

- a random reference point $P_{r e f}$ is chosen among the subset of the workspace $\Gamma_{s u b}$ as the robot total length $L$ is higher than $30 \mathrm{~mm}$. This is useful to analyze the configurations in which the three tubes of the robots are deployed (3 links are obtained),

- the point cloud is scanned in order to find all the reachable points $P_{k} \in \Gamma_{s u b}$ in a defined neighborhood $R$ of that reference such that $\left\|P_{k}-P_{r e f}\right\| \leq R$ (e.g. at $R=1 \mathrm{~mm}$ distance from the reference), - among these points, the end-effector orientations $O_{k}$ are clustered to find the similar ones $\| O_{k}-$ 
$O_{r e f} \| \leq \delta$ (e.g. absolute angle difference lower than $\delta=10^{\circ}$ ),

- the arc parameters $\chi$ of these poses are saved and reinserted in the direct geometric model to display the robot pose.

An example of such numerical simulations is depicted in Figure 10. The superimposition of the robot poses are plotted when the end-effector reaches a neighborhood $(R)$ of a random target position $\left(P_{r e f}\right)$ among the workspace subset point cloud $\Gamma_{s u b}$.
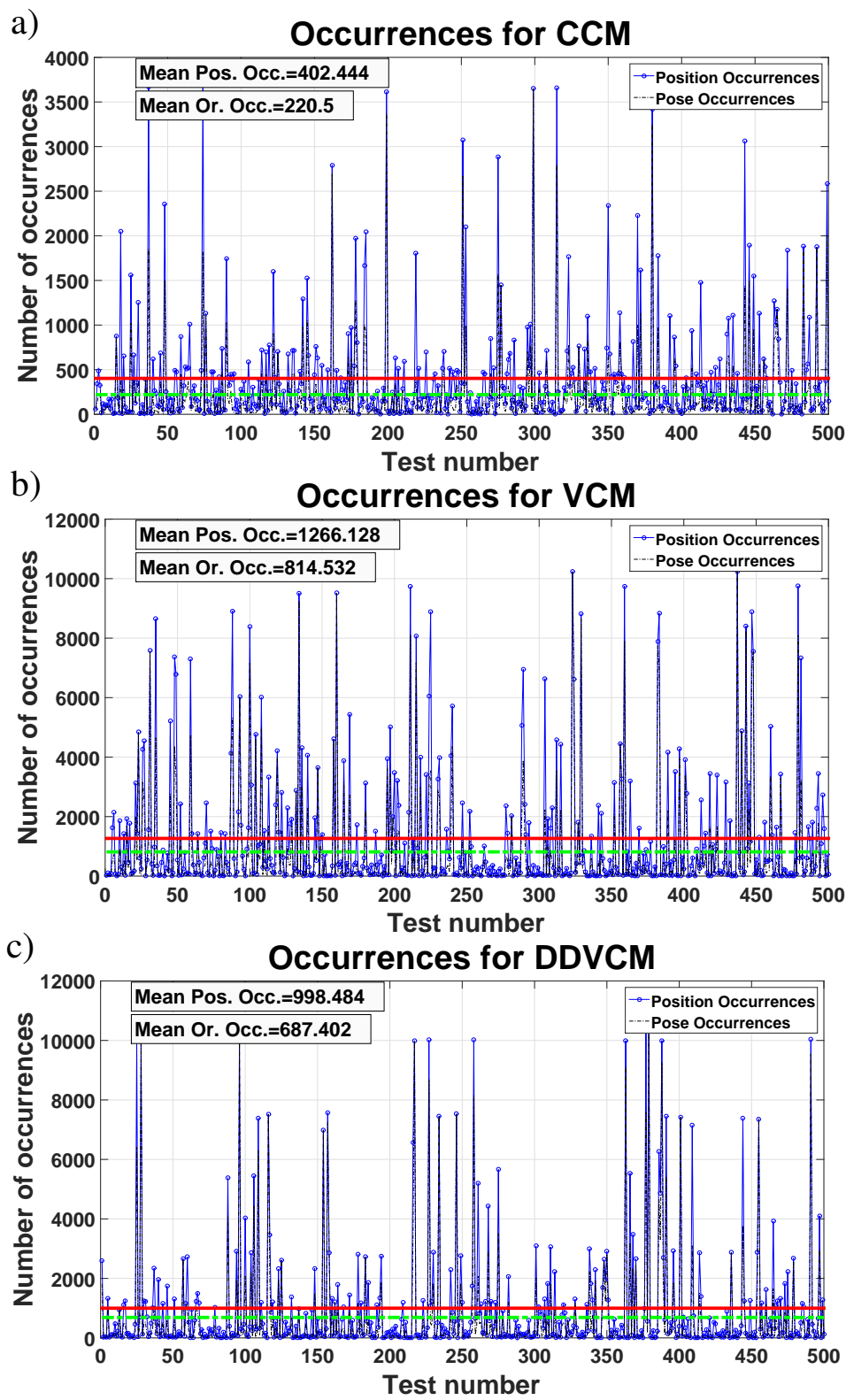

Figure 11: Position and pose occurrences at 500 random points of $\Gamma_{\text {sub }}$ at $L>30 \mathrm{~mm}$ (to ensure three tube extension) among the workspace and at $R=1 \mathrm{~mm}$ distance and $\delta=10^{\circ}$ deviation a) for CCM, b) for VCM, and c) for DDVCM. The solid red line denotes the mean of position occurrences and the dashed green line denotes the mean of pose occurrences. 
The tests are performed 500 times to demonstrate the number of occurrences that appear in the studied models. Results are displayed in Figure 11 showing the number of occurrences in terms of positions. It shows as well the occurrences in terms of orientations among the position occurrences, designated by pose occurrences.

Figure 11 shows that position occurrences are identified more often in VCM and DDVCM than in CCM. The mean amounts are almost two to three times as many than that of CCM. The same analysis is valid for the full pose occurrences. The latter are three to four times higher for VCM and DDVCM than for CCM. It ensues that a target point can be reached with different poses. Moreover, the continuum robot with embedded soft micro-actuation can reach almost a same pose cluster with several actuator configurations more often than CCM. It permits to describe different admissible paths and different robot configurations while aiming the same point and apprehending with the same orientation. Consequently, a reconfigurability is provided to the system. It might also refer to a redundancy ability or a sort of density in terms of pose completion.

\subsection{Performance analysis}

\subsubsection{Kinematic Jacobian matrices and singular configurations}

For the workspace studied herein, singular configurations are detected for the three models in different proportions. Analyzing Table 4, CCM and VCM appear to present the most numerous singular poses with a kinematic Jacobian matrix rank varying mostly between 5 and 6 . In contrast, the DDVCM presents more than $92 \%$ of non-singular configurations out of 262.144 poses. A threshold of $10^{-5}$ is set onto the singular values used by Matlab in order to calculate the matrix rank.

Table 4: Percentages of kinematic Jacobian rank values according to the models.

\begin{tabular}{l||c|c|c|c|c|c}
$\operatorname{rank}\left(\mathbf{J}_{\text {Robot }}\right)$ & $\mathbf{1}$ & $\mathbf{2}$ & $\mathbf{3}$ & $\mathbf{4}$ & $\mathbf{5}$ & $\mathbf{6}$ \\
\hline \hline $\mathbf{C C M}$ & 0 & 0 & 0 & $\simeq 1.5 \%$ & $\simeq 14.3 \%$ & $\simeq 84.1 \%$ \\
\hline $\mathbf{V C M}$ & 0 & 0 & 0 & $\simeq 4.1 \%$ & $\simeq 59.8 \%$ & $\simeq 36.1 \%$ \\
\hline DDVCM & 0 & 0 & 0 & $\simeq 1.5 \%$ & $\simeq 6.2 \%$ & $\simeq 92.2 \%$
\end{tabular}

\subsubsection{Performance indices}

In order to obtain a different meaning of the kinematics of the discussed models, let us assume that $J_{\text {Robot }}$ can be decomposed into a translation velocity kinematic Jacobian $J_{v}$ and a rotation velocity kinematic Jacobian $J_{\omega}$ such that:

$$
\mathbf{J}_{\text {Robot }}=\left[\begin{array}{l}
\mathbf{J}_{v} \\
\mathbf{J}_{\omega}
\end{array}\right]
$$

Following the decomposition of the kinematic Jacobian of equation 37, the singular value decomposition (SVD) is performed to $\mathbf{J}_{v}$ and $\mathbf{J}_{\omega}$ separately such that $\sigma_{i}^{t}$ correspond to $\mathbf{J}_{v}$ singular values and $\sigma_{i}^{r}$ to those of $\mathbf{J}_{\omega}$, where $i=1,2,3$. The translation performances can be illustrated through the manipulability vectors which lengths correspond to $\operatorname{sqrt}\left(\sigma_{i}^{v}\right)$. These vectors describe the ability (or easiness) of the manipulator to move along each direction as shown in Figure 12. The rotation 
performances are described in terms of arcs of circles which lengths are proportional to $\operatorname{sqrt}\left(\sigma_{i}^{\omega}\right)$. The arc lengths are thus proportional to the ability of the robot to rotate around every different direction. The illustration of this manipulability is depicted in Figure 13 for an arbitrary end-effector position of the robot considering the three models studied in this paper.

a)

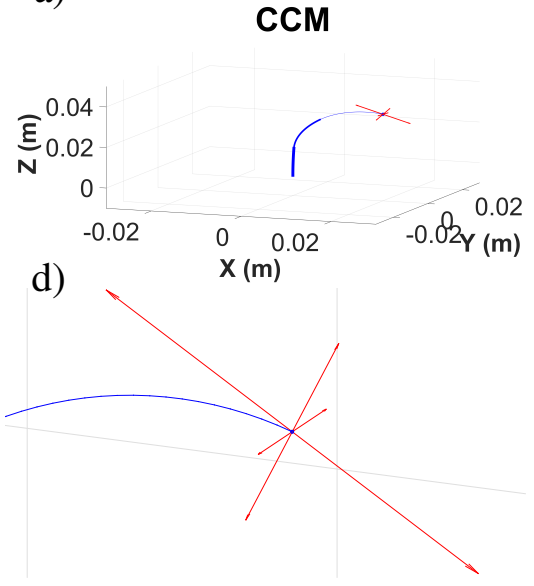

b)

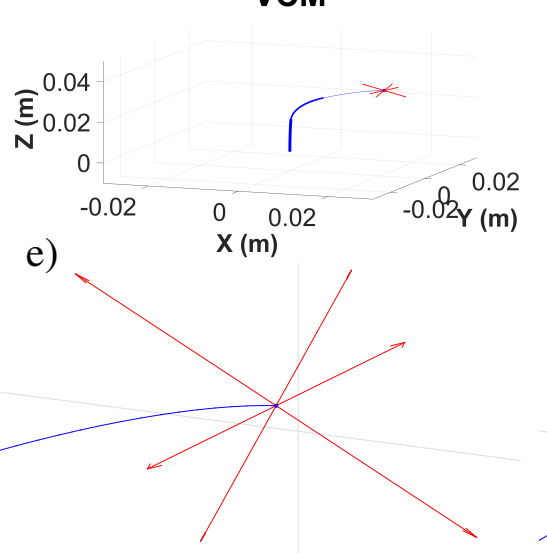

c)

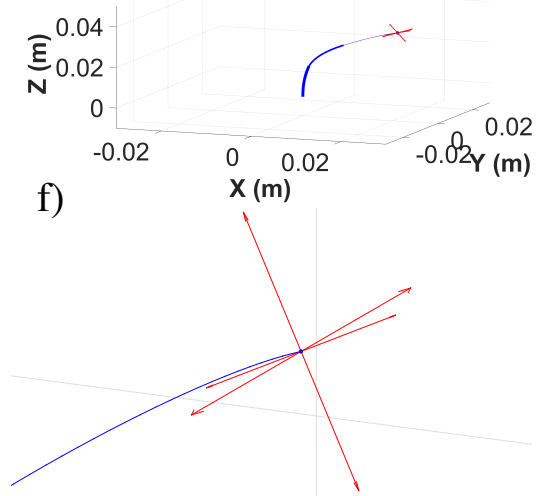

Figure 12: Representation of translational manipulabilities at a same arbitrary end-effector position of the robot for a) CCM, b) VCM, and c) DDVCM. The zoomed figures for d) CCM, e) VCM, and f) DDVCM display the robot in blue and the translation manipulabilities in red vectors.

Figures 12c,f prove that the DDVCM presents the most isotopic behavior with vectors of almost the same length. The robot presents nearly the similar easiness to move along the three different directions presented hereby, in contrast with the disproportionate vectors of CCM and VCM (Figure 12d,c). Figure 13 displays the manipulability in terms of rotation abilities of the manipulator at the same position. Although the CCM shows two rotation directions of almost the same rate (c.f. Figures 13a,d), DDVCM displays the highest rate on two rotation directions (c.f. Figures 13c,f). One can note that one rotational direction is almost forbidden in all the cases, which is due to the robot construction that prevents the rotation in that particular direction.

In order to strengthen the results discussed above, performance indices are employed. Their definitions and computations were introduced, among others, by Yoshikawa (1985a,b); Klein and Blaho (1987); Angeles and López-Cajún (1992). The performance indices ensue from the SVD of the full kinematic Jacobian $J_{\text {Robot }}$. The three most significant performance indices, namely manipulability (M), isotropy (Iso), and condition number inverse (CNI), are defined as follows:

$$
\mathrm{M}=\sqrt{\prod_{i} \sigma_{i}}, \quad \text { Iso }=\frac{\prod_{i} \sigma_{i}}{\sum_{i} \sigma_{i}}, \quad \text { and } \mathrm{CNI}=\frac{\sigma_{\min }}{\sigma_{\max }}
$$

where $\sigma_{i}$ are the singular values of the robot kinematic Jacobian $J_{\text {Robot }}$, M denotes the manipulability, I the isotropy, and CNI the condition number inverse. For each performance index, only singular values corresponding to the maximum rank will be taken into account (e.g. if $\operatorname{rank}\left(\mathbf{J}_{\text {Robot }}\right)=5$, only the first 5 singular values will be employed in the calculus). 
a)

\section{CCM}

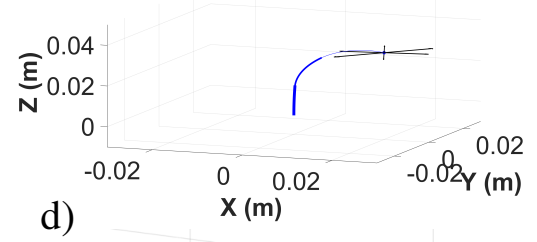

d)

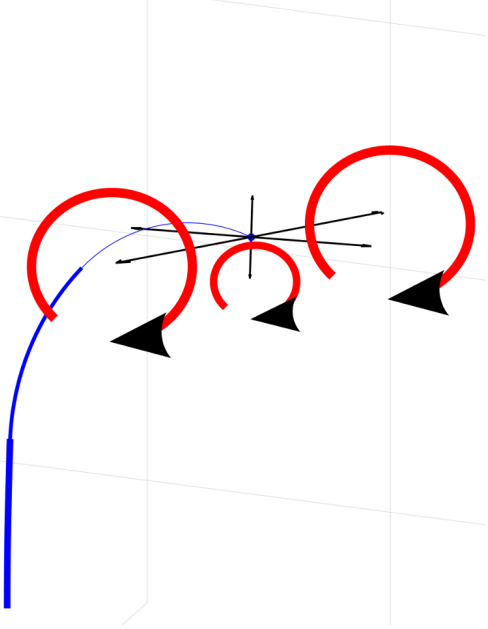

b)

VCM

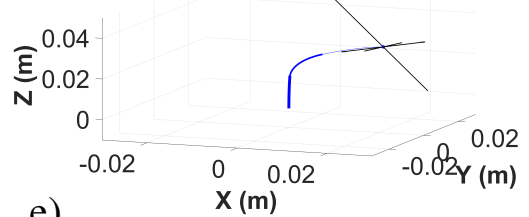

e)

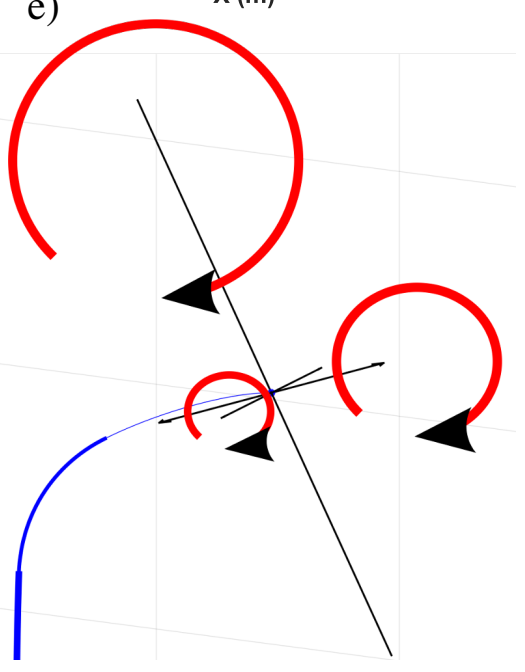

c)
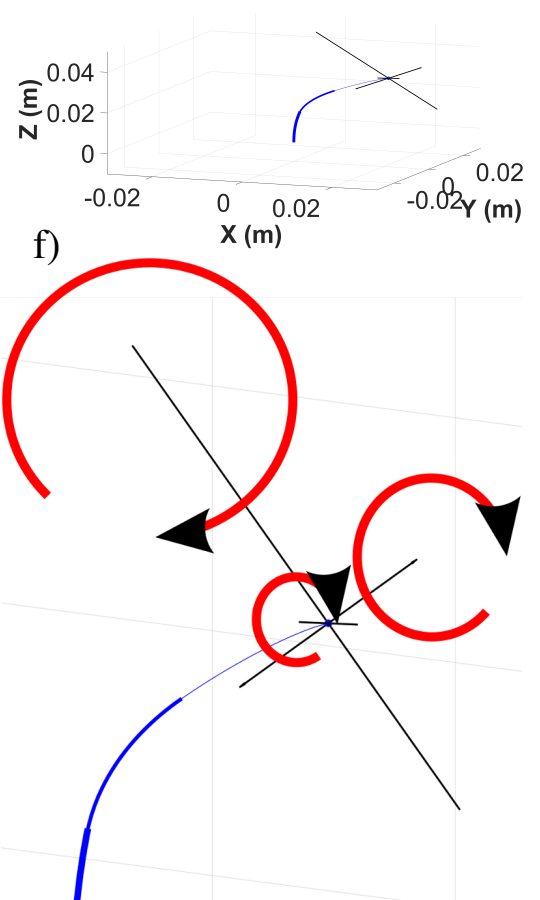

Figure 13: Representation of rotational manipulabilities at a same arbitrary end-effector position of the robot for a) CCM, b) VCM, and c) DDVCM. The zoomed figures for d) CCM, e) VCM, and f) DDVCM display the robot in blue and the rotation manipulabilities in black vectors with the proportionate circle arrow sizes.

Performance indices are depicted in Figures 14, 15, and 16 for CCM, VCM, and DDVCM respectively. Following the same sampling explained in the workspace generation (cf. section 3.1), the performance indices are computed at each point of the cluster (denoted pose index). In terms of increasing pose index, the first values are related to the nearest points to the robot base while the last values correspond to those where the robot is totally deployed. The highest "periodic" values on Figures 14-16 are associated to the configurations of maximum insertion angles for CCM, and maximum curvatures for VCM and DDVCM.

We assume that the global performance indices can be written as follows:

$$
G M=\frac{\int M}{\vartheta}, \quad \text { GIso }=\frac{\int I s o}{\vartheta}, \quad G C N I=\frac{\int C N I}{\vartheta}
$$

where $G M$ is the global manipulability, GIso is the global isotropy, GCNI denotes the global condition number inverse, and $\vartheta$ is the covered volume. The red lines in Figures 14,15, and 16 display these values. 
a)

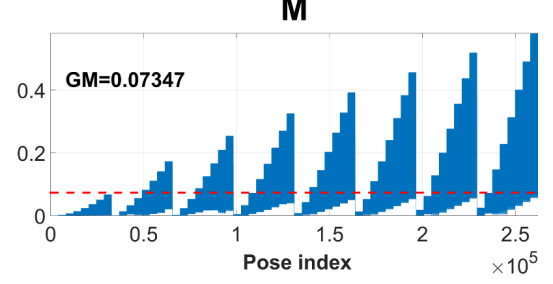

b)

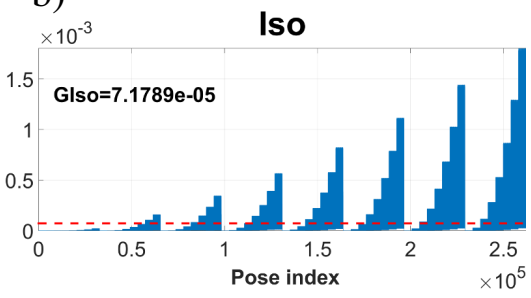

c)

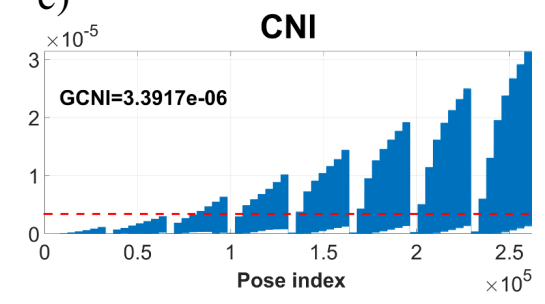

Figure 14: CCM performance index variation with respect to the pose index in the workspace: a) manipulability, b) isotropy, and c) condition number inverse.

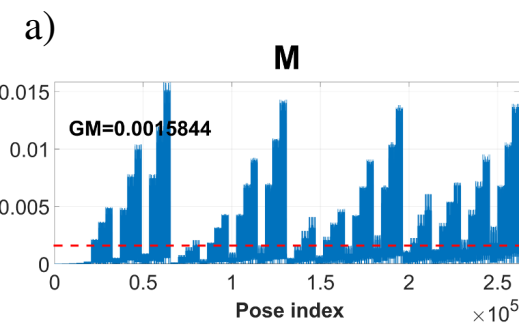

b)

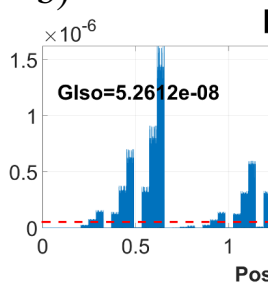

Iso

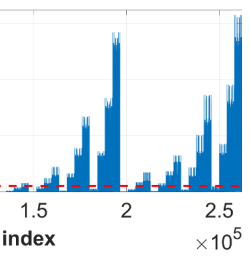

c)

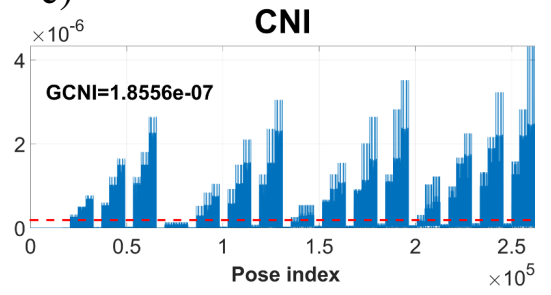

Figure 15: VCM performance index variation with respect to the pose index in the workspace: a) manipulability, b) isotropy, and c) condition number inverse.

a)

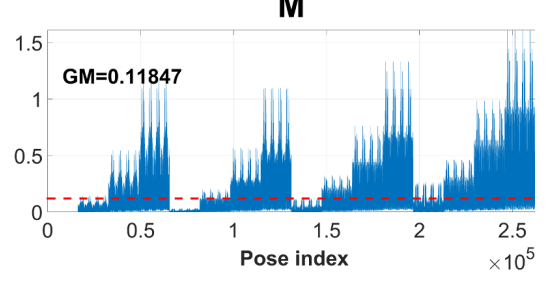

b)

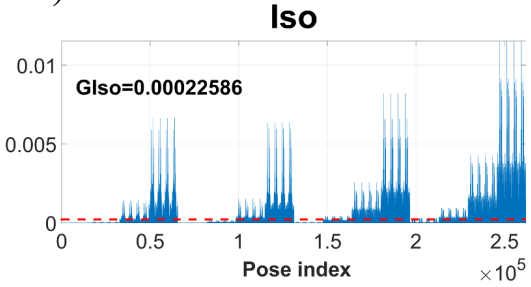

c)

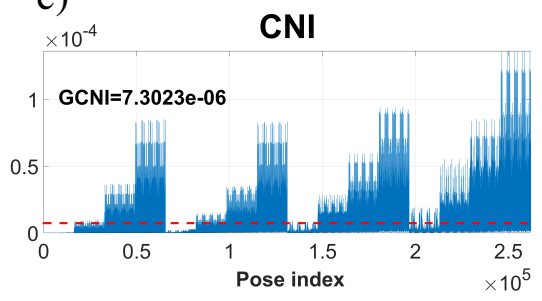

Figure 16: DDVCM performance index variation with respect to the pose index in the workspace: a) manipulability, b) isotropy, and c) condition number inverse.

The performance indices are clearly higher for DDVCM than for CCM and VCM. The manipulability of DDVCM is twice higher than that of CCM. The former is able to translate and rotate along different directions with an enhanced homogeneity. The kinematic Jacobian $\mathbf{J}_{D D V C M}$ is significantly better conditioned than $\mathbf{J}_{C C M}$ and $\mathbf{J}_{V C M}$. Hence fewer singular configurations are detected for DDVCM than for CCM. Thus, these results induce a higher level of safety of the system with DDVCM.

\section{Conclusion}

\subsection{Contributions summary}

A homogeneous formalism was developed to describe three models of concentric tube robots with constant curvature (CCM) and with variable curvature according one and two directions 
(VCM and DDVCM respectively). This generic modeling is useful to clearly identify the advantages of embedding micro-actuation on concentric tubes. In particular, the DDVCM should enable a control of all the arc parameters. The use of the rotation motors can be avoided. Thus, the actuator sizes can be decreased to permit a higher integrability in the OR. It is also expected to provide an additional ease to include the developed robot in a classical endoscopic device as an auxiliary tool. Concerning the kinematics of the studied structures, holonomy is provided when using the VCM and DDVCM as all the arc parameter components can be controlled. Besides, redundancy is brought when using more than three tubes for VCM and DDVCM. In a dimensional purpose, the numerical results prove that the workspace is broadened when bending the tubes beyond a certain value. That is possible with the usage of the embedded micro-actuation in VCM and DDVCM. In terms of position occurrences, the VCM and DDVCM provide more robot configurations to reach the target volume. Added to that, redundancy is provided to the system. In fact, more numerous actuator configurations and robot postures permit the VCM and DDVCM to describe a particular pose than for CCM. Using performance indices, we prove that the homogeneity of the translation and rotation velocity vectors provide the DDVCM with the most isotropic behavior compared to CCM and VCM. In fact, the global performance indices show that DDVCM presents the best conditioned Jacobian matrix and the best manipulability. Thus, the level of safety of the manipulator is improved with the DDVCM. All in all, the DDVCM shows the best features in terms of safety, redundancy and pose occurrences compared to CCM and VCM.

\subsection{Future works}

Many topics related to continuum manipulators need further investigations. The experimental validation of the EAP-based embedded micro-actuators is under development. Additional efforts demand to be supplied to find the best balance between accurate modeling and short computation time. Moreover, including supplementary mechanical constraints as torsion and friction permits an exhaustive description. However, a full mechanically compliant model would slow down the computation speed. For real time environment, we might develop numerical solutions to account for the model complexity while performing on-line control. Our efforts are also focused on the development of a control strategy that includes the novel embedded micro-actuation for concentric tube robots. Finally, all these developments should take into account the medical environment constraints. One major concern is to account for the external loads on the robot body to permit a conformation with the realistic conditions, namely the contact with the surrounding tissue. Such models were introduced for the standard concentric tube robots by Lock et al. (2010); Rucker et al. (2010a) with forces applied at the end-effector of the robot and by Ha et al. (2015) for distributed forces/moments along one or several tubes of robot. These effects should be addressed in future works for the concentric tube robots with embedded actuation to enhance the accuracy of the model. Another important concern is related to the safety that should be maintained when designing and controlling this novel robotic manipulator.

\section{Appendix}

A brief development of an example of each developed model (CCM, VCM, and DDVCM) is introduced in this section. The developments are based on equation 7 concerning the curvature 
components $\kappa_{x}$ and $\kappa_{y}$. One example of the differentiation of the CCM is carried out here to elucidate the matrix formalism used. For the second link, the active actuators regarding the curvature and the equilibrium angle of the second and third tube involved are $\alpha_{2}$ and $\alpha_{3}$. Thus, the curvature component derivatives are:

$$
\left\{\begin{array}{c}
\dot{\kappa}_{x_{2}}=\frac{1}{E_{2} I_{2}+E_{3} I_{3}}\left(-E_{2} I_{2} \kappa_{i, 2} \sin \alpha_{2} \dot{\alpha}_{2}-E_{3} I_{3} \kappa_{i, 3} \sin \alpha_{3} \dot{\alpha}_{3}\right) \\
\dot{\kappa}_{y_{2}}=\frac{1}{E_{2} I_{2}+E_{3} I_{3}}\left(E_{2} I_{2} \kappa_{i, 2} \cos \alpha_{2} \dot{\alpha}_{2}+E_{3} I_{3} \kappa_{i, 3} \cos \alpha_{3} \dot{\alpha}_{3}\right)
\end{array}\right.
$$

We obtain $\mathbf{B}_{2}$ in equation 13. $\mathbf{B}_{1}$ and $\mathbf{B}_{3}$ are developed in the same way taking into account the number of tubes in each link and their actuators $\alpha_{i}$.

Similarly, for the VCM, and for the second link and taking into account equation $7, v_{2}, v_{3}, \alpha_{2}$ and $\alpha_{3}$ are the active actuators for the second and third tube curvatures and equilibrium angles. Thus, the curvature component derivatives are given by:

$$
\left\{\begin{array}{l}
\dot{\kappa}_{x_{2}}=\frac{1}{E_{2} I_{2}+E_{3} I_{3}}\left(E_{2} I_{2} \cos \alpha_{2} \dot{\kappa}_{i n s, 2}+E_{3} I_{3} \cos \alpha_{3} \dot{\kappa}_{i n s, 3}-E_{2} I_{2} \kappa_{i, 2} \sin \alpha_{2} \dot{\alpha}_{2}-E_{3} I_{3} \kappa_{i, 3} \sin \alpha_{3} \dot{\alpha}_{3}\right) \\
\dot{\kappa}_{y_{2}}=\frac{1}{E_{2} I_{2}+E_{3} I_{3}}\left(E_{2} I_{2} \sin \alpha_{2} \dot{\kappa}_{i n s, 2}+E_{3} I_{3} \sin \alpha_{3} \dot{\kappa}_{i n s, 3}+E_{2} I_{2} \kappa_{i, 2} \cos \alpha_{2} \dot{\alpha}_{2}+E_{3} I_{3} \kappa_{i, 3} \cos \alpha_{3} \dot{\alpha}_{3}\right)
\end{array}\right.
$$

And knowing that $\dot{\kappa}_{i n s, 2}=C_{P P y_{2}} \dot{v}_{2}$ and $\dot{\kappa}_{i n s, 3}=C_{P P y_{3}} \dot{v}_{3}$ from equation 18, this leads to the matrix notation $\mathbf{C}_{2}$ in equation 22. The same derivations were performed for the third and first link, only the number of the involved tubes and their associated actuators change and thus the dimensions of $\mathbf{C}_{1}$ and $\mathbf{C}_{3}$.

Concerning the DDVCM, the curvature components $\kappa_{x}$ and $\kappa_{y}$ can be directly controlled respectively by the actuators $v_{x}$ and $v_{y}$. After obtaining the curvature components for each tube due to the actuation (e.g. equation 27), one must inject these values into the curvature component expressions that ensue from the tube interactions ( $c f$. equation 1). The example of the curvature component derivatives of the second link is developed hereby:

$$
\left\{\begin{array}{c}
\dot{\kappa}_{x_{2}}=\frac{1}{E_{2} I_{2}+E_{3} I_{3}}\left(E_{2} I_{2} \cos \alpha_{2} \dot{\kappa}_{2 i n s}+E_{3} I_{3} \cos \alpha_{3} \dot{\kappa}_{3 i n s}-E_{2} I_{2} \kappa_{2_{i n s}} \dot{\phi}_{2_{i n s}}-E_{3} I_{3} \kappa_{3} \dot{\phi}_{\text {ins }} \dot{\phi}_{3_{\text {ins }}}\right) \\
\dot{\kappa}_{y_{2}}=\frac{1}{E_{2} I_{2}+E_{3} I_{3}}\left(E_{2} I_{2} \sin \alpha_{2} \dot{\kappa}_{2_{\text {ins }}}+E_{3} I_{3} \sin \alpha_{3} \dot{\kappa}_{3 i n s}+E_{2} I_{2} \cos \phi_{2} \dot{\phi}_{2_{i n s}}+E_{3} I_{3} \cos \phi_{3} \dot{\phi}_{3_{\text {ins }}}\right)
\end{array}\right.
$$

where $\dot{\kappa}_{2_{\text {ins }}}=\frac{C_{P y_{2}}}{\sqrt{\kappa_{x_{2}}^{2}+\kappa_{y_{2}}^{2}}} \kappa_{x_{2_{i n s}}} \dot{v}_{x, 2}+\frac{C_{P P y_{2}}}{\sqrt{\kappa_{x_{2}}^{2}+\kappa_{y_{2}}^{2}}} \kappa_{y_{2_{i n s}}} \dot{v}_{y, 2}$,

$\dot{\kappa}_{3_{i n s}}=\frac{C_{P P y_{3}}}{\sqrt{\kappa_{x_{3}}^{2}+\kappa_{y_{3}}^{2}}} \kappa_{x_{3 i n s}} \dot{v}_{x, 3}+\frac{C_{P P y_{3}}}{\sqrt{\kappa_{x_{3 i n s}}^{2}+\kappa_{y_{3}}^{2}}} \kappa_{y_{3 i n s}} \dot{v}_{y, 3}$,

$\dot{\phi}_{2_{\text {ins }}}=-\frac{C_{P P y_{2}}}{1+\kappa_{y_{2 i n s}}^{2}} \kappa_{y_{2_{i n s}}} \dot{v}_{x, 2}+\frac{C_{P P y_{2}}}{1+\kappa_{y_{2 i n s}}^{2}} \kappa_{x_{2_{i n s}}} \dot{v}_{y, 2}$, and

$\dot{\phi}_{3_{\text {ins }}}=-\frac{C_{P P y_{3}}}{1+\kappa_{y_{3}}^{2}} \kappa_{y_{3_{i n s}}} \dot{v}_{x, 3}+\frac{C_{P P y_{3}}}{1+\kappa_{y_{3}}^{2}} \kappa_{x_{3 i n s}} \dot{v}_{y, 3}$.

The matrix notation related to these derivations is denoted $\mathbf{D}_{2}$ as presented in equation 32 . Taking into account the suitable actuators for the tubes interacting in the other links, we can develop the two other matrices $\mathbf{D}_{1}$ and $\mathbf{D}_{3}$. 


\section{Acknowledgments}

This work has been supported by the Labex ACTION project (contract ANR-11-LABX-000101) and by NEMRO project (contract ANR-14-CE17-0013).

\section{References}

Alici, G., Huynh, N., Feb 2007. Performance quantification of conducting polymer actuators for real applications: A microgripping system. IEEE/ASME Transactions on Mechatronics 12 (1), 73-84.

Anderson, V., Horn, R., 1967. Tensor Arm Manipulator Design. American Society of Mechanical Engineers.

Andreff, N., Tamadazte, B., Jul 2015. Laser steering using virtual trifocal visual servoing. The International Journal of Robotics Research 35 (6), 672-694.

Angeles, J., López-Cajún, C. S., 1992. Kinematic isotropy and the conditioning index of serial robotic manipulators. International Journal of Robotics Research 11 (6), 560-571.

Anor, T., Madsen, J. R., Dupont, P. E., Jan 2011. Algorithms for design of continuum robots using the concentric tubes approach: A neurosurgical example. In: IEEE International Conference on Robotics and Automation. Shanghai, China, pp. 667-673.

Baek, C., Yoon, K., Kim, D.-N., Dec 2015. Finite element modeling of concentric-tube continuum robots. Structural Engineering \& Mechanics 5 (57), 809-821.

Bailly, Y., Amirat, Y., Apr 2005. Modeling and control of a hybrid continuum active catheter for aortic aneurysm treatment. In: IEEE International Conference on Robotics and Automation. Barcelona, Spain, pp. 924-929.

Bar-Cohen, Y., 2001. Electroactive Polymer (EAP) Actuators as Artificial Muscles: Reality, Potential, and Challenges. Press Monographs. SPIE Press.

Bedell, C., Lock, J., Gosline, A., Dupont, P. E., Apr 2011. Design optimization of concentric tube robots based on task and anatomical constraints. In: IEEE International Conference on Robotics and Automation. Shanghai, China, pp. 398-403.

Bergeles, C., Dupont, P. E., Nov 2013. Planning stable paths for concentric tube robots. In: IEEE/RSJ International Conference on Intelligent Robots and Systems. Tokoy, Japan, pp. 30773082.

Burgner, J., Swaney, P., Lathrop, R., Weaver, K., Webster III, R. J., Sep 2013. Debulking from within: A robotic steerable cannula for intracerebral hemorrhage evacuation. IEEE Transactions on Biomedical Engineering 60 (9), 2567-2575. 
Burgner, J., Swaney, P. J., Rucker, D. C., Gilbert, H. B., Nill, S. T., P. T. Russell, I., Weaver, K. D., Webster III, R. J., Sep 2011. A bimanual teleoperated system for endonasal skull base surgery. In: IEEE/RSJ International Conference on Intelligent Robots and Systems. San Francisco, USA, pp. 2517-2523.

Butler, E. J., Hammond-Oakley, R., Chawarski, S., Gosline, A. H., Codd, P., Anor, T., Madsen, J. R., Dupont, P. E., Lock, J., Oct 2012. Robotic neuro-endoscope with concentric tube augmentation. In: IEEE/RSJ International Conference on Intelligent Robots and Systems. Vilamoura, Portugal, pp. 2941-2946.

Camarillo, D. B., Milne, C. F., Carlson, C. R., Zinn, M. R., Salisbury, J. K., 2008. Mechanics modeling of tendon-driven continuum manipulators. IEEE Transactions on Robotics 24 (6), $1262-1273$.

Chen, G., 2005. Design, modeling and control of a micro-robotic tip for colonoscopy. Ph.D. thesis, INSA, Vileurbanne, France.

Chikhaoui, M. T., Rabenorosoa, K., Andreff, N., 2014a. Kinematic modeling of an EAP actuated continuum robot for active micro-endoscopy. In: Lenarčič, J., Khatib, O. (Eds.), Advances in Robot Kinematics. Springer International Publishing, pp. 457-465.

Chikhaoui, M. T., Rabenorosoa, K., Andreff, N., December 2014b. Towards clinical application of continuum active micro-endoscope robot based on EAP actuation. In: Surgetica. Chambéry, France.

Chirikjian, G., Jul 1993. A continuum approach to hyper-redundant manipulator dynamics. In: IEEE/RSJ International Conference on Intelligent Robots and Systems. Vol. 2. Tokyo, Japan, pp. 1059-1066.

Chirikjian, G., Burdick, J., Jun 1994. A modal approach to hyper-redundant manipulator kinematics. IEEE Transactions on Robotics and Automation 10 (3), 343-354.

Choi, H., Ryew, S., Jung, K., Kim, H., Jeon, J., Nam, J., Maeda, R., Tanie, K., 2002. Microrobot actuated by soft actuators based on dielectric elastomer. In: IEEE/RSJ International Conference on Intelligent Robots and Systems. Vol. 2. Lausanne, Switzerland, pp. 1730-1735.

Cianchetti, M., Ranzani, T., Gerboni, G., De Falco, I., Laschi, C., Menciassi, A., Nov 2013. Stiffflop surgical manipulator: Mechanical design and experimental characterization of the single module. In: IEEE/RSJ International Conference on Intelligent Robots and Systems. pp. 35763581 .

Ciéslak, R., Morecki, A., Jan 1999. Elephant trunk type elastic manipulator - a tool for bulk and liquid materials transportation. Robotica, 11-16.

Comber, D. B., Cardona, D., Webster III, R. J., Barth, E. J., 2012. Sliding mode control of an mricompatible pneumatically actuated robot. In: ASME Symposium on Fluid Power and Motion Control. Cambridge, USA, pp. 283-293. 
Dehghani, M., Moosavian, S., July 2011. Modeling and control of a planar continuum robot. In: IEEE/ASME International Conference on Advanced Intelligent Mechatronics. pp. 966-971.

Dehghani, M., Moosavian, S. A. A., Feb 2013. Modeling of continuum robots with twisted tendon actuation systems. In: RSI/ISM International Conference on Robotics and Mechatronics. Tehran, Iran, pp. 14-19.

Dupont, P., Gosline, A., Vasilyev, N., Lock, J., Butler, E., Folk, C., Cohen, A., Chen, R., Schmitz, G., Ren, H., et al., 2012a. Concentric tube robots for minimally invasive surgery. In: Hamlyn Symposium on Medical Robotics. Vol. 7. p. 8.

Dupont, P. E., Chawarski, S., Butler, E. J., Hammond-Oakley, R., Gosline, A. H., Codd, P., Anor, T., Madsen, J. R., Lock, J., 2012b. Extending the reach and stability of manually steerable neuroendoscopes through robotics. In: Hamlyn Symposium on Medical Robotics. pp. 89-90.

Dupont, P. E., Lock, J., Itkowitz, B., Butler, E., Apr 2010. Design and control of concentric-tube robots. IEEE Transactions on Robotics 26 (2), 209-225.

Gravagne, I., Walker, I., Apr 2000a. Kinematic transformations for remotely-actuated planar continuum robots. In: IEEE International Conference on Robotics and Automation. Vol. 1. San Francisco, USA, pp. 19-26.

Gravagne, I., Walker, I. D., 2000b. Kinematics for Constrained Continuum Robots Using Wavelet Decomposition. American Society of Civil Engineers, Ch. 37, pp. 292-298.

Gravagne, I. A., Rahn, C. D., Walker, I. D., Jun 2003. Large deflection dynamics and control for planar continuum robots. IEEE/ASME Transactions on Mechatronics 8 (2), 299-307.

Gravagne, I. A., Walker, I. D., Oct 2001. Manipulability and force ellipsoids for continuum robot manipulators. In: IEEE/RSJ International Conference on Intelligent Robots and Systems. Vol. 1. Maui, USA, pp. 304-311.

Gravagne, I. A., Walker, I. D., Jun 2002. Manipulability, force, and compliance analysis for planar continuum manipulators. IEEE Transactions on Robotics 18 (3), 263-273.

Ha, J., Park, F., Dupont, P., 2015. Elastic stability of concentric tube robots subject to external loads. IEEE Transactions on Biomedical Engineering PP (99), 1-1.

Haga, Y., Mineta, T., Makishi, W., Matsunaga, T., Esashi, M., 2010. Shape Memory Alloys. InTech, Ch. Active Bending Catheter and Electric Endoscope Using Shape Memory Alloy, pp. 107-127.

Hannan, M. W., Walker, I. D., 2003. Kinematics and the implementation of an elephant's trunk manipulator and other continuum style robots. Journal of Robotic Systems 20 (2), 45-63.

Hendrick, R. J., Herrell, S., Webster III, R. J., May 2014. A multi-arm hand-held robotic system for transurethral laser prostate surgery. In: IEEE International Conference on Robotics and Automation. Hong Kong, China, pp. 2850-2855. 
Hirose, S., 1993. Biologically inspired robots : snake-like locomotors and manipulators / Shigeo Hirose ; translated by Peter Cave and Charles Goulden. Oxford University Press Oxford ; New York.

Ikeuchi, M., Ikuta, K., 2009. Development of pressure-driven micro active catheter using membrane micro emboss following excimer laser ablation (meme-x) process. In: IEEE International Conference on Robotics and Automation. Kobe, Japan, pp. 4469-4472.

Ikuta, K., Tsukamoto, M., Hirose, S., Apr 1988. Shape memory alloy servo actuator system with electric resistance feedback and application for active endoscope. In: IEEE International Conference on Robotics and Automation. Vol. 1. Philadelphia, USA, pp. 427-430.

Jones, B., Gray, R., Turlapati, K., Oct 2009. Three dimensional statics for continuum robotics. In: IEEE/RSJ International Conference on Intelligent Robots and Systems. Saint Louis, USA, pp. 2659-2664.

Jones, B., Walker, I., Feb 2006a. Kinematics for multisection continuum robots. IEEE Transactions on Robotics 22 (1), 43-55.

Jones, B. A., Walker, I. D., Apr 2005. A new approach to jacobian formulation for a class of multi-section continuum robots. In: IEEE International Conference on Robotics and Automation. Barcelona, Spain, pp. 3268-3273.

Jones, B. A., Walker, I. D., Dec 2006b. Practical kinematics for real-time implementation of continuum robots. IEEE Transactions on Robotics 22 (6), 1087-1099.

Kim, K. J., Tadokoro, S., 2007. Electroactive polymers for robotic applications. Springer, springerVerlag London.

Klein, C. A., Blaho, B. E., Jul. 1987. Dexterity measures for the design and control of kinematically redundant manipulators. International Journal of Robotic Research 6 (2), 72-83.

Langelaar, M., van Keulen, F., Apr 2004. Modeling of a shape memory alloy active catheter. In: AIAA Structures, Structural Dynamics and Materials Conference. Palm Springs, USA, pp. 1922.

Lee, K. K. C., Munce, N. R., Shoa, T., Charron, L. G., Wright, G. A., Madden, J. D., Yang, V. X. D., 2009. Fabrication and characterization of laser-micromachined polypyrrole-based artificial muscle actuated catheters. Sensors and Actuators A-Physical 153 (2), 230-236.

Lee, S.-K., Lee, S.-J., An, H.-J., Cha, S.-E., Chang, J. K., Kim, B., Pak, J. J., 2002. Biomedical applications of electroactive polymers and shape-memory alloys. SPIE Smart Structures and Materials 4695, 17-31.

Lock, J., Laing, G., Mahvash, M., Dupont, P. E., Oct 2010. Quasistatic modeling of concentric tube robots with external loads. In: IEEE/RSJ International Conference on Intelligent Robots and Systems. Taipei, Taiwan, pp. 2325-2332. 
Murray, R. M., Li, Z. L., Sastry, S. S., 1994. A Mathematical Introduction to Robotic Manipulation. CRC Press.

Otake, M., Kagami, Y., Inaba, M., Inoue, H., 2002. Motion design of a starfish-shaped gel robot made of electro-active polymer gel. Robotics and Autonomous Systems 40 (23), 185 - 191.

Robinson, G., Davies, J., May 1999. Continuum robots - a state of the art. In: IEEE International Conference on Robotics and Automation. Vol. 4. Detroit, USA, pp. 2849-2854.

Rone, W. S., Ben-Tzvi, P., Jun 2014. Mechanics modeling of multisegment rod-driven continuum robots. Journal of Mechanisms and Robotics 6 (4), 12.

Rucker, D., Webster III, R. J., Dec 2011. Statics and dynamics of continuum robots with general tendon routing and external loading. IEEE Transactions on Robotics 27 (6), 1033-1044.

Rucker, D. C., Jones, B. A., Webster III, R. J., Aug 2010a. A geometrically exact model for externally loaded concentric-tube continuum robots. IEEE Transactions on Robotics 26 (5), 769-780.

Rucker, D. C., Webster III, R. J., Chirikjian, G. S., Cowan, N. J., Sep 2010b. Equilibrium conformations of concentric-tube continuum robots. International Journal of Robotics Research 29 (10), 1263-1280.

Schneider, J. S., Burgner, J., Webster III, R. J., Russell III, P. T., 2013. Robotic surgery for the sinuses and skull base: what are the possibilities and what are the obstacles? Current opinion in otolaryngology \& head and neck surgery 21 (1), 11-16.

Sears, P., Dupont, P., Oct 2006. A steerable needle technology using curved concentric tubes. In: IEEE/RSJ International Conference on Intelligent Robots and Systems. Beijing, China, pp. 2850-2856.

Sears, P., Dupont, P. E., Apr 2007. Inverse kinematics of concentric tube steerable needles. In: IEEE International Conference on Robotics and Automation. Roma, Italy, pp. 1887-1892.

Shoa, T., Madden, J., Fekri, N., Munce, N., Yang, V. X., Aug 2008. Conducting polymer based active catheter for minimally invasive interventions inside arteries. In: IEEE International Conference Engineering in Medicine and Biology Society. pp. 2063-2066.

Shoa, T., Madden, J. D., Munce, N. R., Yang, V., 2010. Analytical modeling of a conducting polymer-driven catheter. Polymer International 59 (3), 343-351.

Simaan, N., Taylor, R., Flint, P., Sep 2004. Medical Image Computing and Computer-Assisted Intervention Conference. Springer Berlin Heidelberg, Saint-Malo, France, Ch. High Dexterity Snake-Like Robotic Slaves for Minimally Invasive Telesurgery of the Upper Airway, pp. 17-24.

Torres, L. G., Webster III, R. J., Alterovitz, R., Oct 2012. Task-oriented design of concentric tube robots using mechanics-based models. In: IEEE/RSJ International Conference on Intelligent Robots and Systems. Vilamoura, Portugal, pp. 4449-4455. 
Trivedi, D., Rahn, C. D., Kier, W. M., Walker, I. D., 2008. Soft robotics: Biological inspiration, state of the art, and future research. Applied Bionics and Biomechanics 5 (3), 99-117.

Walker, I., Hannan, M., Sep 1999. A novel ‘elephant's trunk’ robot. In: IEEE/ASME International Conference on Advanced Intelligent Mechatronics. Atlanta, USA, pp. 410-415.

Webster III, R. J., 2007. Design and mechanism of continuum robot for surgery. Ph.D. thesis, The John Hopkins University, USA.

Webster III, R. J., Jones, B. A., 2010. Design and kinematic modeling of constant curvature continuum robots: A review. International Journal of Robotics Research 29 (13), 1661-1683.

Webster III, R. J., Kim, J. S., Cowan, N. J., Chirikjian, G. S., Okamura, A. M., May $2006 a$. Nonholonomic modeling of needle steering. International Journal of Robotics Research 25 (56), 509-525.

Webster III, R. J., Okamura, A. M., Cowan, N. J., Oct 2006b. Toward active cannulas: Miniature snake-like surgical robots. In: IEEE/RSJ International Conference on Intelligent Robots and Systems. Beijing, China, pp. 2857-2863.

Webster III, R. J., Okamura, A. M., Cowan, N. J., Taylor, R. H., Jan 2013. Active cannula for bio-sensing and surgical intervention. US Patent App. 13/416,772.

Webster III, R. J., Romano, J. M., Cowan, N. J., May 2008. Kinematics and calibration of active cannulas. In: IEEE International Conference on Robotics and Automation. Pasadena, USA, pp. 3888-3895.

Webster III, R. J., Romano, J. M., Cowan, N. J., Feb 2009. Mechanics of precurved-tube continuum robots. IEEE Transactions on Robotics 25 (1), 67-78.

Wolf, A., Brown, H., Casciola, R., Costa, A., Schwerin, M., Shamas, E., Choset, H., Oct 2003. A mobile hyper redundant mechanism for search and rescue tasks. In: IEEE/RSJ International Conference on Intelligent Robots and Systems. Vol. 3. Tokyo, Japan, pp. 2889-2895.

Xu, K., Simaan, N., Jun 2008. An investigation of the intrinsic force sensing capabilities of continuum robots. IEEE Transactions on Robotics 24 (3), 576-587.

Yoshikawa, T., Mar 1985a. Manipulability and redundancy control of robotic mechanisms. In: IEEE International Conference on Robotics and Automation. Vol. 2. Saint Louis, USA, pp. 1004-1009.

Yoshikawa, T., 1985b. Manipulability of robotic mechanisms. International Journal of Robotics Research 4 (2), 3-9. 